\title{
Experimental disagreements with extended unitarity
}

\author{
D.V. Bugg ${ }^{\mathrm{a}}$ \\ Queen Mary, University of London, Mile End Rd., London E1 4NS, UK
}

Received: 12 December 2007 /

Published online: 19 January 2008 - (c) Springer-Verlag / Società Italiana di Fisica 2008

\begin{abstract}
In production processes, e.g. $J / \Psi \rightarrow \omega \pi \pi$ or $\bar{p} p \rightarrow 3 \pi$, the $\sigma$ and $f_{0}(980)$ overlap in the same partial wave. The conjecture of extended unitarity (EU) states that the $\pi \pi$ pair should have the same phase variation as $\pi \pi$ elastic scattering. This is an extension of Watson's theorem beyond its original derivation, which stated only that the $s$-dependence of a single resonance should be universal. The prediction of EU is that the deep dip observed in $\pi \pi$ elastic scattering close to $1 \mathrm{GeV}$ should also appear in production data. Four sets of data disagree with this prediction. All require different relative magnitudes of $\sigma$ and $f_{0}(980)$. That being so, a fresh conjecture is to rewrite the 2-body unitarity relation for production in terms of observed magnitudes. This leads to a prediction different to EU. Central production data from the AFS experiment fit naturally to this hypothesis.
\end{abstract}

PACS. 13.25.-k; 13.25.Gv; 13.75.Lb

\section{Introduction}

In its simplest form, the idea of extended unitarity (EU) states that the $\pi \pi$ pair in a single partial wave should have the same phase variation with $s$ in all reactions as in elastic scattering. This idea originates from Aitchison [1] and has been adopted in various guises by many authors. His arguments will be presented in detail in Sect. 2, so as to expose the assumptions and consequences. At the time the idea was introduced, it was a reasonable conjecture; now modern data allow it to be checked accurately, but disagree with it.

Many experimental groups have made extensive fits to production data using a $K$-matrix approach based on EU. These fits are excellent; no criticism is intended of their quality. Experimentalists have found empirically the necessary freedom to get good fits to data. However, on close inspection, this freedom is inconsistent with strict EU.

This whole topic has been the subject of extensive discussion with many authors. There is a bewildering jungle of claims and counter-claims. My objective is to cut a path through this tangle and expose where problems lie; this makes the presentation pedantic in places.

Aitchison's essential point is that all processes should be described by a universal denominator $[1-\mathrm{i} \rho(s) K(s)]$, where $K$ is the same as for elastic scattering; $\rho$ is Lorentz invariant phase space. The assumption which is being made is that Watson's theorem [2] applies to the coherent sum of all components in the $J^{P}=0^{+}$partial wave. This is a step beyond Watson's derivation, which referred only

\footnotetext{
a e-mail: D.Bugg@rl.ac.uk
}

to a single eigenstate; Watson did not consider overlapping resonances.

In $\pi \pi$ elastic scattering, the $f_{0}(980)$ is superimposed on a slowly rising amplitude associated with the $\sigma$ pole. CernMunich data [3] show that the phases of these two components add. Below the $K K$ threshold, both $\sigma$ and $f_{0}(980)$ $T$-matrices $T=\mathrm{e}^{\mathrm{i} \delta} \sin \delta$ are confined to the unitarity circle if we neglect the tiny inelasticity due to $\pi \pi \rightarrow \gamma \gamma$. Unitarity may be satisfied by multiplying $S$-matrices $S=\mathrm{e}^{2 \mathrm{i} \delta}$ of $\sigma$ and $f_{0}(980)$, as suggested by Dalitz and Tuan [4]. This fits the data within errors of $\sim 3.5^{\circ}$.

Figure 1 shows the Argand diagram for the $I=0 \pi \pi$ $S$-wave from my recent re-analysis of these (and other) data [5]. From BES data on $J / \Psi \rightarrow \phi \pi^{+} \pi^{-}$, the $f_{0}(980)$ has a full-width at half-maximum of $34 \pm 8 \mathrm{MeV}$ [6]. The combined phase shift rises rapidly from $90^{\circ}$ at $0.88 \mathrm{GeV}$ to $270^{\circ}$ near $1.1 \mathrm{GeV}$. There is a deep dip in the cross section where the combined phase goes rapidly through $180^{\circ}$. The crucial point of EU is that this feature should be common to production processes.

BES data on $J / \Psi \rightarrow \phi \pi \pi$ [6] immediately require a modification of the rudimentary form of EU. The $\pi \pi$ mass spectrum in these data is reproduced in Fig. 2. There is a dominant $f_{0}(980)$ contribution and a small interfering $\sigma$ contribution; this is very different to elastic scattering. Lähde and Meissner [7] modify the conjecture of EU to apply separately to strange and non-strange components, i.e. to the scalar form factors for $\pi \pi$ and $K K$.

The dip in the elastic cross section at $989 \mathrm{MeV}$ is a very delicate feature. If, for any reason, relative magnitudes of $\sigma$ and $f_{0}$ change, the zero at $989 \mathrm{MeV}$ can disappear quickly; here and elsewhere, $f_{0}$ will denote $f_{0}(980)$ unless there is 


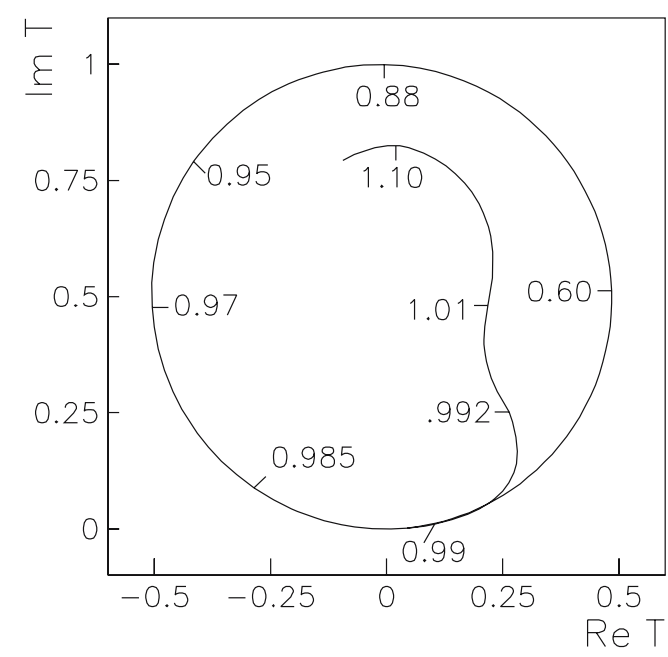

Fig. 1. Argand plot of the $\pi \pi I=0 S$-wave in elastic scattering; masses are marked in $\mathrm{GeV}$

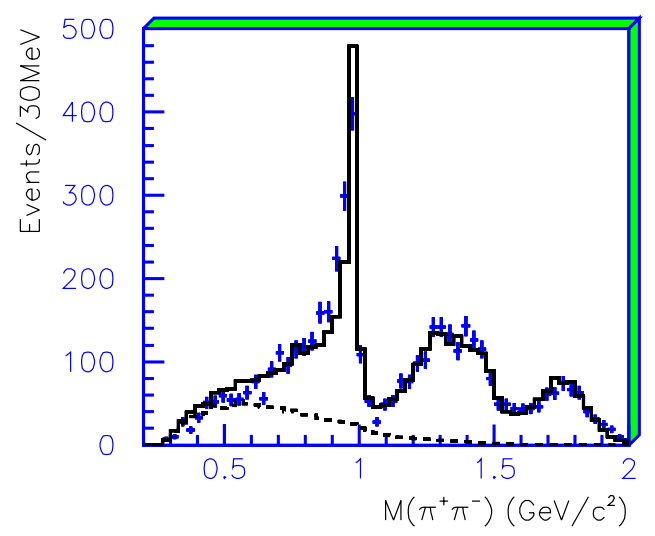

Fig. 2. The $\pi \pi$ mass projection for BES data on $J / \Psi \rightarrow$ $\phi \pi^{+} \pi^{-}$: the upper histogram shows the current fit to experimental points [5]; the lower histogram shows the fitted $\sigma$ component

confusion with other $f_{0}$ 's. If the phase of $f_{0}$ changes with respect to the $\sigma$, the mass at which the dip appears will likewise change. The interference region between $\sigma$ and $f_{0}(980)$ is an ideal place to check extended unitarity.

Two considerations will play a critical role: unitarity and analyticity. Unitarity is often quoted and plays an essential role in setting up the current $K$-matrix formalism which treats both elastic scattering and production on the same basis. For a production reaction, Aitchison conjectures a unitarity relation for the production amplitude $F$ :

$$
\operatorname{Im} F=F T_{\mathrm{el}}^{*} .
$$

He defines his $F$ to be proportional to $T^{(p)} / \rho$, where $T^{(p)}$ refers to production:

$$
F \rho=\alpha T^{(p)} .
$$

Dividing both sides of (1) by $\alpha$,

$$
\operatorname{Im} T^{(p)}=T^{(p)} T_{\mathrm{el}}^{*} .
$$

It is odd that $T^{(p)}$ on the right-hand side is multiplied by $T_{\mathrm{el}}^{*}$, unless $T^{(p)}=T_{\mathrm{el}}$. However, experiment will require different contributions to $T^{(p)}$ from $\sigma$ and $f_{0}$.

Consider next analyticity. Dispersion relations connect magnitudes and phases. If the relative magnitudes of $f_{0}$ and $\sigma$ change from those of elastic scattering because of matrix elements, their relative phases must also change. Conversely, analyticity predicts that if the phase variation with $s$ of the amplitude is universal, as EU demands, so is the variation with $s$ of the magnitude (up to a constant scaling factor); for the simplest situation where only resonances are present, the relative magnitudes of $\sigma, f_{0}(980)$ and any further $f_{0}$ must be almost the same in production as elastic scattering. This is a point which has almost always been ignored.

The word 'almost' represents a caveat: there may in addition be a polynomial is $s$ which can be different between elastic scattering and production. It turns out that one can plausibly limit deviations of relative magnitudes within $12 \%$. This question is discussed in Sect. 2.1. Experiment requires larger deviations than this in the four sets of data discussed here. This implies phases must change from those predicted by EU. Experimentalists have correctly allowed for this by using complex coupling constants in the isobar model.

Section 3 compares the prediction of EU with 3 sets of data. The first concerns BES data for $J / \Psi \rightarrow \omega \pi^{+} \pi^{-}$[8]. The non-strange components of $\sigma$ and $f_{0}$ dominate both elastic scattering and $J / \Psi \rightarrow \omega \pi \pi$. From Aitchison's algebra and that of Lähde and Meissner, it follows that the $f_{0}$ amplitude should have almost the same magnitude as the $\sigma$ amplitude, as well as the same phase as elastic scattering. This prediction is contradicted by the data, where no $f_{0}(980)$ is visible and a fit to the data places a low limit on it.

The next two sets are Crystal Barrel data for $\bar{p} p \rightarrow 3 \pi^{0}$, where $\sigma$ and $f_{0}(980)$ are clearly visible [9]. One set is for annihilation in liquid hydrogen and the other for gaseous hydrogen. Annihilation from the ${ }^{3} P_{1}$ initial state is $13 \%$ in liquid and $48 \%$ in gas, allowing a clear separation of amplitudes for production of $\sigma$ and $f_{0}$ from ${ }^{1} S_{0}$ and ${ }^{3} P_{1}$. Results for both are inconsistent with the deep dip of elastic scattering predicted by EU.

Section 4 concerns data from the AFS experiment on central production: $p p \rightarrow p p \pi^{+} \pi^{-}[10]$. Here one expects the protons in the final state to act as spectators. However, EU still fails conspicuously to fit the data. This important result leads to a revised form of the unitarity relation, as follows.

Figure 3 sketches the usual diagrammatic approach to the unitarity relation. It may be derived by cutting the diagram down the middle, along the dashed line. For a 2-body system of $\pi \pi, K K, \eta \eta$, etc. the resulting relation is well known:

$$
\operatorname{Im} T_{\mathrm{el}}=T_{\mathrm{el}} T_{\mathrm{el}}^{*} .
$$

The application of 2-body unitarity assumes that the pions interact only with one another, not with any spectator. In most sets of data there are large signals where pions do in- 


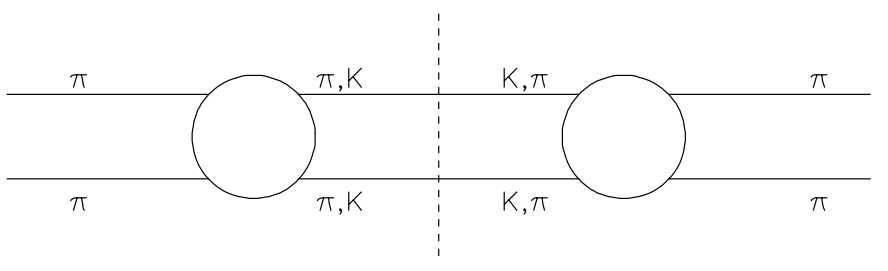

Fig. 3. Unitarity diagram for $\pi \pi \rightarrow \pi \pi$

teract with the spectator. For $J / \Psi \rightarrow \omega \pi \pi$, as an example, the $b_{1}(1235) \pi$ channel accounts for $40 \%$ of events [8]. Some of it may be generated by pions from decays of $\sigma$ or $f_{0}$ rescattering from the spectator; this is a so-called triangle graph. Aitchison himself remarks that this can distort the unitarity relation. This provides one reason why EU may fail for the first three sets of data; it does not explain the fourth, where some further effect is required.

There are fundamental differences between elastic scattering and production. In $J / \Psi \rightarrow \omega \pi \pi$, for example, matrix elements $\langle J / \Psi \mid \omega \sigma\rangle$ and $\left\langle J / \Psi \mid \omega f_{0}\right\rangle$ dictate the magnitudes of these amplitudes; any values are possible. This differs from elastic scattering, where $\sigma$ and $f_{0}$ magnitudes are fixed purely by their coupling constants $g_{\pi}$ to $\pi \pi$. Equation (3) is an asymmetric relation, allowing $\sigma$ and $f_{0}$ to be produced with different magnitudes, but requiring that they rescatter an in elastic scattering. A more logical alternative is the symmetric relation

$$
\operatorname{Im} T^{(p)}=T^{(p)} T^{(p) *},
$$

hence $\operatorname{Im} F=F T^{*(p)}$. This relation fits AFS data for central production naturally, whereas EU does not. If the $f_{0}$ is absent from production data, (5) reduces to the obvious relation $\operatorname{Im} T^{\sigma}=\left|T^{\sigma}\right|^{2}$; Aitchison's form of the relation, taken with analyticity does not allow the $f_{0}$ to be absent, as we shall see in Sect. 2 .

Section 5 suggests a new way of fitting 2-body data. Section 6 then summarises conclusions.

\section{The hypothesis of extended unitarity}

In a two-body process, the scattering of a pair of pions to final states $\pi \pi, K K, \eta \eta, 4 \pi$ and $\gamma \gamma$ must satisfy unitarity. The $T$-matrix for these coupled channels may be written in terms of a real $K$-matrix as

$$
T_{\mathrm{el}}=\rho K(1-\mathrm{i} \rho K)^{-1} .
$$

It is normalised here so that $T_{\pi \pi}=\left(\eta \mathrm{e}^{2 \mathrm{i} \delta}-1\right) / 2 \mathrm{i}$. Below the inelastic threshold

$$
\rho K=\tan \delta .
$$

The $T$-matrices used here will include couplings to all channels. However, it simplifies the presentation of essential points to reduce the formalism initially to a single $\pi \pi$ channel. This simplication is sufficient to expose the basic issues, and can be generalised later to include inelasticity.
The approach of Aitchison [1] will now be outlined. I am grateful to him for clarifying the algebra in more detail than is to be found in the original publication. Suppose $S$-matrices multiply, i.e. phases add. Let $K_{A}$ and $K_{B}$ be $K$-matrices for $\sigma$ and $f_{0}$ respectively. The elementary expression for $\tan \left(\delta_{A}+\delta_{B}\right)$ then gives a $K$-matrix for elastic scattering

$$
K_{\mathrm{el}}=\frac{K_{A}+K_{B}}{1-\rho^{2} K_{A} K_{B}}
$$

from which it follows that the $T$-matrix for elastic scattering is

$$
T_{\mathrm{el}}=\frac{\left(K_{A}+K_{B}\right) \rho}{\left(1-\mathrm{i} \rho K_{A}\right)\left(1-\mathrm{i} \rho K_{B}\right)} .
$$

Aitchison now conjectures that an amplitude $F$ for producing a two-body channel present in $K_{\mathrm{el}}$ may be written in terms of a vector $P$, with

$$
\begin{aligned}
& F=\left(1-\mathrm{i} \rho K_{\mathrm{el}}\right)^{-1} P, \\
& P=\frac{\alpha_{A} K_{A}+\alpha_{B} K_{B}}{1-\rho^{2} K_{A} K_{B}},
\end{aligned}
$$

where $\alpha_{A}$ and $\alpha_{B}$ are constants for production couplings. With this ansatz, the relation

$$
\operatorname{Im} F=F T_{\mathrm{el}}^{*},
$$

known as extended unitarity, is automatically satisfied. It is a consequence of (10) that $F$ has the same phase as $T_{\mathrm{el}}$. Substituting (8) and (11) in (10) gives, in this one-channel case

$$
\begin{aligned}
F & =\frac{\alpha_{A} K_{A}+\alpha_{B} K_{B}}{\left(1-\mathrm{i} \rho K_{A}\right)\left(1-\mathrm{i} \rho K_{B}\right)}, \\
& =\alpha_{A}\left[T_{A}\left(1+\mathrm{i} T_{B}\right)+\beta T_{B}\left(1+\mathrm{i} T_{A}\right)\right] / \rho,
\end{aligned}
$$

where $\beta=\alpha_{B} / \alpha_{A}$. From (13), the phase of $F$ is indeed $\delta_{A}+$ $\delta_{B}$, as imposed by (10). Equation (14) will play the decisive role in comparisons with experiment.

In (14), $T_{A}\left(1+\mathrm{i} T_{B}\right)=\exp \mathrm{i}\left(\delta_{A}+\delta_{B}\right) \sin \delta_{A} \cos \delta_{B}$ and $T_{B}\left(1+\mathrm{i} T_{A}\right)=\operatorname{expi}\left(\delta_{A}+\delta_{B}\right) \cos \delta_{A} \sin \delta_{B}$. At $989 \mathrm{MeV}$, $\delta_{f}=90^{\circ}$ and $\delta_{\sigma}=92^{\circ}$. So both terms are very close to zero, regardless of the values of $\alpha_{A}$ and $\alpha_{B}$. This predicts that production data should have the same deep dip at this energy as elastic scattering.

There is a further point. In the second term, $\left(1+\mathrm{i} T_{A}\right) \simeq$ 0 over a sizable mass range. In the first term, $T_{B}$ should be conspicuous, since it has a rapid phase variation and the same peak magnitude as $T_{A}$, which is itself clearly visible in all sets of data considered here. However, the data all require the magnitude of the $f_{0}(980)$ to be smaller than predicted.

The key point is that the factor $1 /\left(1-\mathrm{i} \rho K_{B}\right)$ of $(13)$ leads directly to the factor $\left(1+\mathrm{i} T_{B}\right)$ in the first term of (14). The first and third sets of data will require $\beta$ of (14) to be small. In the elastic region, the first term becomes

$$
F \simeq \mathrm{i} \alpha_{A}\left(1+\mathrm{i} e^{\mathrm{i} \delta_{B}} \sin \delta_{B}\right) .
$$


The bizarre conclusion of $\mathrm{EU}$ is that the phase of the $f_{0}(980)$ is present even though $\alpha_{B} \simeq 0$. This is inconsistent with analyticity. It will be shown in Sect. 3 that experiment disagrees with EU even without the constraint of analyticity. However this additional constraint makes conclusions more definitive.

\subsection{Analyticity}

For purely elastic scattering, the Omnès relation [11] reads (including a factor $\rho(s)$ in $N(s)$ ):

$$
\begin{aligned}
T_{\mathrm{el}}(s) & =N(s) / D(s), \\
D(s) & =\mathrm{e}^{-\mathrm{i} \delta(s)} \exp -\left[\frac{s-4 m_{\pi}^{2}}{\pi} \mathrm{P} \int \frac{\mathrm{d} s^{\prime}}{s^{\prime}-4 m_{\pi}^{2}} \frac{\delta\left(s^{\prime}\right)}{s-s^{\prime}}\right]
\end{aligned}
$$

where $\mathrm{P}$ denotes the principal value integral. We shall not actually need to evaluate (16). It plays only a conceptual role and this needs considerable explanation. The basic point is that $D(s)$ contains both real and imaginary parts, so $\delta(s)$ determines both. For elastic scattering $N(s)$ is real. It arises from the left-hand cut, i.e. meson exchanges between the two pions.

With inelasticity, corresponding relations may be written in a 2-channel form. Then $\delta(s)$ is replaced by $\phi(s)$, the angle $T$ makes to the real axis when measured from the origin of the Argand diagram, see Fig. 12 of Sect. 4.1.

If $\mathrm{EU}$ is valid, the production amplitude may be written $F=X(s) / D(s)$. In principle $X(s)$ could be anything, depending on production dynamics. However, we have quite precise experimental information about it. An extreme view is that $\alpha_{A}$ and $\alpha_{B}$ of (13) can be arbitrary and complex. However if they are complex this leads directly to a conflict with EU. Equation (14) contains two parts $T_{A}\left(1+\mathrm{i} T_{B}\right)$ and $T_{B}\left(1+\mathrm{i} T_{A}\right)$. Substituting BreitWigner formulae for $T_{A}$ and $T_{B}$, the first term becomes $g_{A}^{2}\left(M_{B}^{2}-s\right) / D_{A}(s) D_{B}(s)$. This is real but has a specific $s$-dependence in the numerator as well as in the denominator. If $\alpha_{A}$ or $\alpha_{B}$ becomes complex, the numerator becomes complex. This then introduces a phase variation separate from $D(s)$. The "prediction" of EU is distorted by this extra phase. Only if $X(s)$ is real does EU survive in its strict form.

Many experimental groups have used the $P$-vector approach using complex coupling coefficients, without realising that this destroys the universality of the phase variation with $s$. This is what experiment demands, so they have done the right thing. But the use of the universal denominator $[1-\mathrm{i} \rho K(\mathrm{el})]$ is no longer logically correct. One might as well fit directly in terms of complex coupling constants and individual $T$-matrices for each resonance.

\subsection{Form factors}

There is a further fundamental point. For elastic scattering, $N(\mathrm{el})$ is uniquely related to $\operatorname{Im} D(s)$ by both unitarity and analyticity. At first sight it appears that analyticity relates $X(s)$ in the same way to $D(s)$ in production reactions, with the result $X(s)=\alpha N(\mathrm{el})$, where $\alpha$ is a constant. This requires $\beta=1$ : if the phase of the $\pi \pi$ amplitude is universal, relative magnitudes of $\sigma$ and $f_{0}$ must also be universal.

There is however a caveat. A more fundamental form of the unitarity relation (12) is that the discontinuity of $F$ across the elastic branch cut is $2 \mathrm{i} F T^{*}$. Then $F$ may be multiplied by a polynomial $X(s)$, providing it does not have a discontinuity along the real $s$-axis. A few examples will hopefully clarify ideas. Firstly, a form factor in $s$ is one such example, arising from the sizes of particles, i.e. from matrix elements. Secondly, in $\phi \rightarrow \gamma f_{0}$, the E1 transition has an intensity proportional to the cube of the photon momentum; this inflates the lower side of the $f_{0}(980)$. Thirdly, in ${ }^{3} P_{1} \bar{p} p \rightarrow \pi^{0} \sigma$, there is an $L=1$ centrifugal barrier for the production process. Fourthly, in some special cases, matrix elements may go through zero as a function of $s$. Taking $X(s)$ to be real, let us write in general

$$
F=X(s) N(\mathrm{el}) / D(s) .
$$

A feature of all production data considered here is a strong low-mass $\pi \pi$ peak due to the $\sigma$ pole. This peak is not present in elastic scattering because of an Adler zero in the elastic scattering amplitude at $s_{A} \simeq 0.41 \mathrm{~m}_{\pi}^{2}$, just below the $\pi \pi$ threshold. The elastic amplitude rises approximately linearly with $s$ and there is no low mass peak. The origin of the difference has been known to theorists for at least 20 years. Au, Morgan and Pennington [12] pointed out that the difference between elastic scattering and central production data can be accomodated by using the same Breit-Wigner denominator for both, but replacing the numerator $N(\mathrm{el})$ by something close to a constant. This polynomial is allowed because $s_{A}$ is outside the physical region. Data require $X(s) N(\mathrm{el}) \simeq 1$, hence $X(s) \simeq 1 / N(\mathrm{el}) \simeq 1 /\left(s-s_{A}\right)$. More exactly,

$$
X(s)=1 /\left[\left(s-s_{A}\right)(1+b s) \exp \left[-\left(s-M_{A}^{2}\right) / A\right]\right.
$$

for the parametrisation of the $\sigma$ amplitude in [13]. In practice, quadatic and cubic terms in $s$ are very small and under tight control from fits to data up to $1.8 \mathrm{GeV}$.

For $J / \Psi \rightarrow \omega \pi \pi$, the $\sigma$ pole is visible by eye in Fig. 4a below. The phase of the $\sigma$ amplitude in this reaction is experimentally the same as in elastic scattering within $\sim 3.5^{\circ}$ [14]. Values of $N(\operatorname{prodn})=X(s) N(\mathrm{el})$ can be determined directly from the data. The same is true of the $\kappa[15]$, which likewise has an Adler zero in the numerator for elastic scattering, but not for production. In both cases, $N($ prodn) is consistent within errors with a constant; the Adler zero in the numerator of elastic scattering has disappeared. One can try fitting the $\sigma$ and $\kappa$ poles in production data with the conventional form factor $N(\operatorname{prodn})=$ $\exp \left(-k^{2} R^{2} / 6\right)$, where $k$ is momentum in the production channel. For both, $R^{2}$ optimises at slightly negative values, which are unphysical. For the $\sigma, R^{2}<0.30 \mathrm{fm}^{2}$ with $95 \%$ confidence and for the $\kappa, R^{2}<0.38 \mathrm{fm}^{2}$ at the same level.

A crucial piece of information in testing $\mathrm{EU}$ will be relative magnitudes of $\sigma$ and $f_{0}$. The magnitude of the $\sigma$ amplitude is easily separated from the tail of the $f_{0}(980)$ at $920 \mathrm{MeV}$, three half-widths from $989 \mathrm{MeV}$. If $X(s)$ is determined at this energy and at $400 \mathrm{MeV}$, the $\sigma$ amplitude 

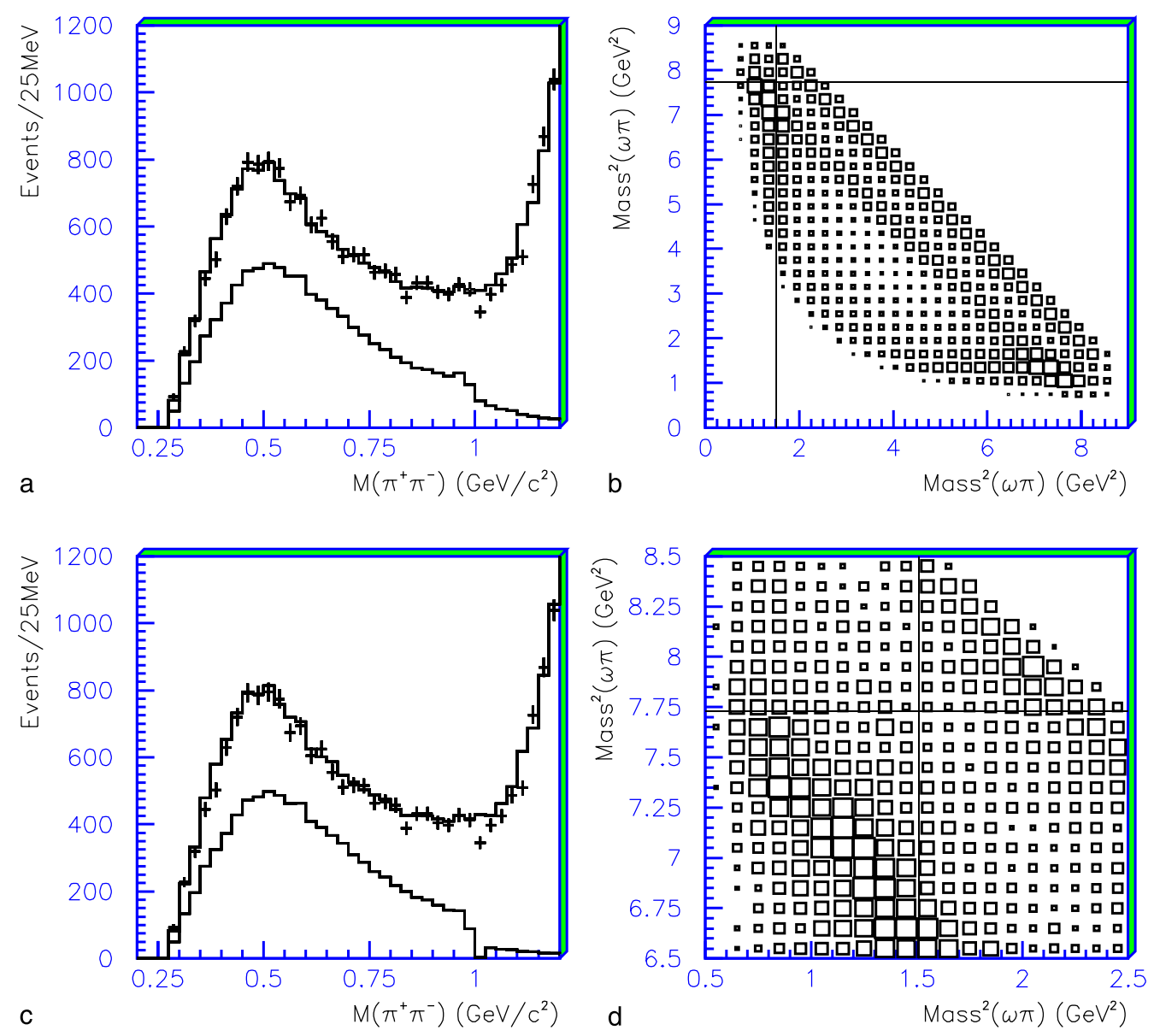

Fig. 4. Fits to BES data for $J / \psi \rightarrow \omega \pi^{+} \pi^{-}:$a $\pi \pi$ mass projection from the full Dalitz plot using the $\sigma$ amplitude of [13]; the lower histogram shows the $S$-wave component; b Dalitz plot from data; $\mathbf{c}$ as a using (14) for EU; d enlarged view of part of the Dalitz plot changes by $20 \%$ at $989 \mathrm{MeV}$ for the $95 \%$ confidence level quoted above. However, this exaggerates the error, since there are compensating changes in the line-shape fitted to the amplitude. Realistically, changes are half this. Adding in quadrature uncertainties in the $\sigma$ line-shape due to the opening of the $K K$ threshold, the uncertainty in the amplitude extrapolated from 920 to $989 \mathrm{MeV}$ is $<12 \%$ with $95 \%$ confidence. This provides a tight constraint on the relative magnitudes of $\sigma$ and $f_{0}(980)$ amplitudes if EU is correct. This disposes of the paradox that the phase of $f_{0}(980)$ can be present with $\alpha_{B}=0$.

From this point onwards, it will be assumed that EU should be supplemented with the condition imposed by analyticity within $12 \%$.

\subsection{Formulae for $\sigma$ and $f_{0}$}

Formulae and numerical parameters for the $\sigma$ amplitude are to be found in [13] and [5]. The $\pi \pi$ coupling has been fitted to four sources: (i) phase shifts deduced from CernMunich data by Ochs [16], (ii) $K_{e 4}$ data of Pislak et al. [17], (iii) predictions of $\pi \pi$ phase shifts by Caprini et al. [18] using the Roy equations, and (iv) BES data on $J / \Psi \rightarrow$ $\omega \pi^{+} \pi^{-}[8]$.

The coupling to $K K$ and $\eta \eta$ has been fitted to available data on those channels [19], and in [5] the $4 \pi$ coupling has been derived from Cern-Munich data. There is close consistency between all these sets of data. Because [13] and [5] fit the same data with different formulae, the amplitudes agree within errors up to 1.2 . Those of [5] are more cumbersome to use, since they allow for the dispersive effect of the $4 \pi$ threshold. Therefore the first and fourth sets of data discussed below are fitted with the formulae from [13].

The general procedure adopted here is to allow the parameters of the $\sigma$ to have the freedom allowed in earlier determinations of its parameters, but no extra freedom in the vicinity of $f_{0}(980)$. In testing EU, the magnitude of $f_{0}$ is restricted to the $12 \%$ discussed above; its phase is allowed the freedom with which its parameters are known.

\subsection{Parameters of $f_{0}(980)$}

The $f_{0}(980)$ is so narrow that it is readily separated from the $\sigma$. The BES data on $J / \Psi \rightarrow \phi \pi^{+} \pi^{-}$shown in Fig. 2 exhibit a very clear $f_{0}(980) \rightarrow \pi \pi$ signal. An important point is the excellent mass resolution and mass calibration of the BES detector, $\sim 4 \mathrm{MeV}$. Both are easily checked for the $K K$ channel against the very precisely known parameters of $\phi(1020)$. Data from the same publication [6] on $\phi K^{+} K^{-}$ contain a clear $f_{0}(980) \rightarrow K K$ peak, and the two sets of data determine accurately the ratio $g^{2}(K K) / g^{2}(\pi \pi)$ of couplings to $K K$ and $\pi \pi$. An important detail is an error in 
units in [6]: $g^{2}(\pi \pi)$ is reported as $165 \mathrm{MeV}$; this should read $0.165 \mathrm{GeV}^{2}$.

The Breit-Wigner denominator for the $f_{0}(980)$ amplitude is $\left[M^{2}-s-\mathrm{i}\left(g_{1}^{2} \rho_{1}+g_{2}^{2} \rho_{2}\right)\right]$ and $\rho_{2}$ has to be continued analytically below the $K K$ threshold as $\mathrm{i} \sqrt{4 m_{K}^{2} / s-1}$. Without direct information on the $K K$ channel, this term gets confused with $\left(M^{2}-s\right)$ [20]. Any form factor applied to $g^{2}(K K)$ adds to the confusion. One only has to glance at the Particle Data Tables [21] to see the large spread in parameters fitted to the $f_{0}(980)$ (and $a_{0}(980)$ ) in experiments having no direct information on the branching ratio between $K K$ and $\pi \pi$. Unfortunately, the PDG does not report the BES determination of $g^{2}(K K) / g^{2}(\pi \pi)$, which is the best in the published literature because of the availability of a clear signal in $\phi K K$.

It was shown in [19] that the BES parameters for $f_{0}(980)$ are closely consistent with Kloe data [22] on $\phi \rightarrow \pi^{0} \pi^{0} \gamma$ when one allows for interference between $\sigma$ and $f_{0}$. This paper determines $g^{2}(\sigma \rightarrow K K) / g^{2}(\sigma \rightarrow \pi \pi)=$ $0.6 \pm 0.1$. BES data on $J / \Psi \rightarrow \omega K^{+} K^{-}$confirm a large value $\geq 0.6$ for this ratio [23]. The $\pi \pi$ full width at halfmaximum $(34 \mathrm{MeV})$ agrees well with Cern-Munich data $30 \pm 10 \mathrm{MeV}$ [3]. It also agrees closely with the full-width of the $f_{0}(980)$ signal in Crystal Barrel data $(\sim 46 \mathrm{MeV})$. The BES parametrisation will therefore be adopted in fitting the AFS data. Further checks from Belle, Babar and Cleo $\mathrm{C}$ will be very welcome.

\section{Experimental tests}

\section{$3.1 J / \Psi \rightarrow \omega \pi \pi$}

Considerable detail needs to be given of fits to experimental data, in order to pin down the disagreements with EU. The prime conclusions which will emerge are that (i) the $\pi \pi$ amplitude does not follow that of elastic scattering, (ii) the magnitude of the $f_{0}(980)$ amplitude, relative to $\sigma$, is much smaller than predicted by (14), regardless of analyticity which also requires that their relative magnitudes should be the same within $12 \%$.

Figure 4 displays features of BES data for $J / \Psi \rightarrow$ $\omega \pi \pi[8]$. There is a clear peak in Fig. 4 a at $\sim 0.5 \mathrm{GeV}$ due to the $\sigma$ pole. Its shape is cleanly separated from the $f_{2}(1270)$ contribution up to $1.05 \mathrm{GeV}$, where the $f_{2}$ rapidly overtakes it. The data include a slowly varying $14 \%$ background which is included in the fit. There is also a well defined slowly varying component due to the reflection of $b_{1}(1235)$. Both are shown in the experimental publication.

There are two amplitudes for production of $\sigma$ and $f_{0}(980)$, with orbital angular momenta $L=0$ and 2 in the production reaction. The $L=2$ amplitude includes a centrifugal barrier for production which optimises at a radius of $0.8 \mathrm{fm}$ (roughly as expected for convolution of wave functions of $\sigma, \omega$ and $f_{0}$ ). For the isobar model fit, different magnitudes are allowed for $L=0$ and 2, with complex coupling constants, though it turns out that the $L=2$ contribution from $f_{0}(980)$ is negligible. In the EU fit the relative phases of $\sigma$ and $f_{0}$ components are constrained to be the same, and relative magnitudes are constrained to be the same within $12 \%$. In Fig. $4 \mathrm{a}$ and c, the lower histogram shows the $J^{P}=0^{+}$intensity: from the isobar model fit in Fig. 4a and from EU in Fig. 4c. The upper histogram, close to data points, shows the result of the full fit. Unfortunately, the mass projection alone is not definitive, for reasons explained shortly. One low point in the mass projection just above $1 \mathrm{GeV}$ hints at a dip following the EU prediction.

However, there is a great deal more information contained in the Dalitz plot of Fig. 4b and also in the correlation between the $\omega$ decay plane and the $\omega \pi \pi$ production plane. In the Dalitz plot, there are strong horizontal and vertical bands due to $b_{1}(1235)$. These bands interfere with $\sigma$ and $f_{2}(1270)$ (and $f_{0}(980)$ if present). Note that the $b_{1}(1235)$ decays mostly through $S$-waves, so its intensity would be almost constant across the Dalitz plot in the absence of interferences. Also note from the lower histogram of Fig. 4a that the $\sigma$ amplitude at $950 \mathrm{MeV}$ is sizable. Cross-hairs on Fig. $4 \mathrm{~b}$ show where a $\pi \pi$ pair of mass $1 \mathrm{GeV}$ intersects the $b_{1}$ band. If $f_{0}(980)$ were present with the same magnitude as $\sigma$ and with the phase predicted by EU, a large but narrow interference with $b_{1}$ cannot be avoided due to $f_{0}(980)$, whatever the relative phase of $b_{1}(1235)$. It should have a full width of $\sim 0.07 \mathrm{GeV}^{2}$ along the $b_{1}$ band: the line-width of $f_{0}(980) \rightarrow \pi \pi$. There should also be a dip somewhere along the diagonal at $m_{\pi \pi}=1 \mathrm{GeV}$. Fig. $4 \mathrm{~d}$ shows an enlarged view of this region. There is no sign of these features.

The $\omega$ decay plays an important role. The spin of the $\omega$ lies along the normal to its decay plane; information on this decay plane is a key ingredient in determining helicity amplitudes. These are essential to determine interferences between the amplitudes for $\omega \sigma, \omega f_{2}(1270)$ and $b_{1}(1235) \pi$. There are in principle five $f_{2}(1270)$ amplitudes corresponding to production with $L=0$ (one amplitude), $L=2$ (three) and $L=4$ (one). It turns out that the $L=4$ amplitude and one of the three $L=2$ amplitudes are negligible. There are large interferences between $\sigma$ and the remaining three $f_{2}(1270)$ amplitudes and even larger interferences with $b_{1}(1235)$.

With the EU hypothesis, the best fit to the Dalitz plot (upper histogram) and the $\omega$ decay plane fills in the predicted dip at $s_{\pi \pi}=1 \mathrm{GeV}^{2}$ with other interferences, see Fig. 4c; however, the price is a considerable deterioration of log likelihood compared with the fit of Fig. 4a. The isobar model fit is better than EU by 259 in log likelihood. There are two reasons. Firstly, the narrow $f_{0}$ does not appear in interferences with either $b_{1}$ or $f_{2}$. The mass resolution of the BES data is $4 \mathrm{MeV}$ in $\pi \pi$ at $950 \mathrm{MeV}$; searching for the $f_{0}(980)$ with accurately known line-shape is limited purely by statistics and there are $\sim 40 \mathrm{~K}$ events. Secondly, in the fit based on EU, there are strong conflicts between interference terms amongst $b_{1}(1235) \pi, \omega f_{2}(1270)$ and $\omega \sigma$. The data want slowly varying interferences between $b_{1}$ and the broad $f_{2}$, instead of rapidly varying interferences of the narrow $f_{0}$ with $b_{1}$ and/or $f_{2}$.

There are two extra fitting parameters for the isobar model fit: i.e. two complex coupling constant for $\omega \sigma$ instead of one. (The $f_{0}(980) \omega L=2$ amplitude is negligible.) 
The definition of log likelihood is such that a change of 0.5 corresponds to a one standard deviation change in one variable. For large statistics, $\chi^{2}$ is approximately twice the change in log likelihood. So the fit of Fig. 4a is better than EU by 19.7 standard deviations statistically.

It is important to remark that the BES publication gives a second fit done independently. This shows a $\pi \pi$ mass projection almost identical with the scalar form factor. This requires a very strong $f_{0}(980)$, in conflict with what one can see by eye in Fig. 4d. However, this analysis made no use of the $\omega$ decay plane. Without that information, it is impossible to disentangle the magnitudes of the five $f_{2}(1270)$ amplitudes; the determination of the magnitude and phase of the $f_{0}(980)$ signal becomes very poor. I have rechecked the analysis omitting information from the $\omega$ decay plane. No stable fit emerges with the $\pi \pi$ mass projection of the second fit in the BES publication, and there is no evidence for the presence of $f_{0}(980)$ at all in this fit. The only explanation I can find of this second BES fit is that the relative magnitudes of $f_{0}(980)$ and $\sigma$ have been constrained to agree with the scalar form factor. This is, of course, exactly what needs to be checked in the present work.

Figure 5 shows the Argand diagram for $T(\operatorname{prodn})=F \rho$ from my fit to $\sigma$ and $f_{0}$; the factor $\rho$ is included so that the diagram goes to zero at the $\pi \pi$ threshold. The ratio of $f_{0}$ and $\sigma$ amplitudes is $0.12 \pm 0.06$ at $0.99 \mathrm{GeV}$ and the $f_{0}$ lags the $\sigma$ by $(42 \pm 20)^{\circ}$. At the top of a large loop due to the $\sigma$, there is a small structure due to $f_{0}(980)$. For comparison purposes, the dashed curve shows the $\sigma$ amplitude alone and the dotted curve shows the EU prediction normalised to the full curve at $0.47 \mathrm{GeV}$, the peak of the $\sigma$. The essential point is that the deep dip in this latter curve near the $f_{0}$ mass is missing from the data. There is one quali-

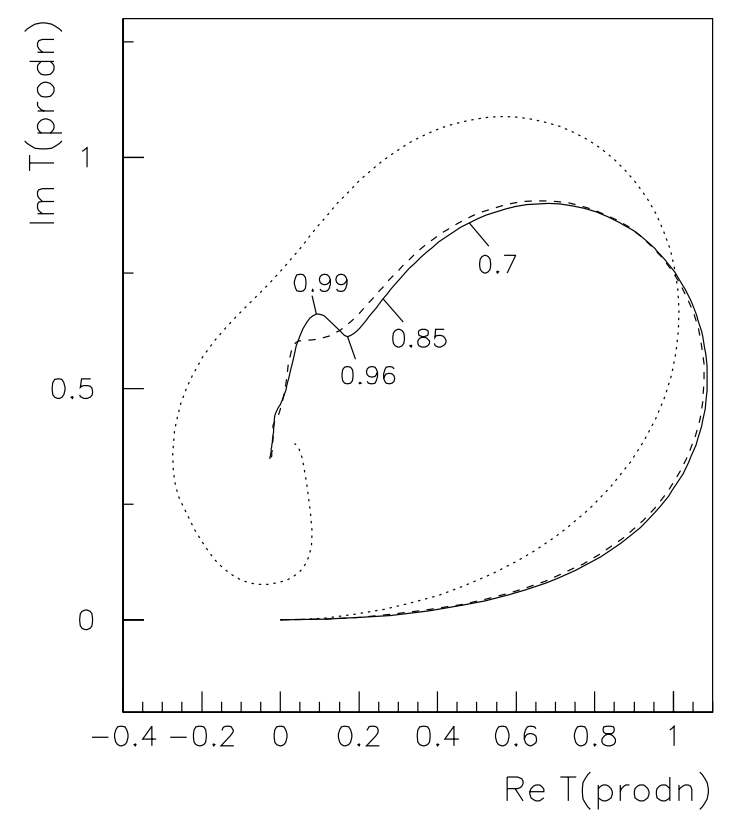

Fig. 5. Argand diagram for $F \rho$; masses are marked in $\mathrm{GeV}$. Full curve, fit; dashed curve, $\sigma$ alone; dotted curve, EU prediction tative feature of Fig. 5 which will be important later. The amplitudes in a production process are free to add vectorially in any way required by the data. This situation is quite different from elastic scattering, where the amplitude is constrained to the unitary circle.

It is now necessary to consider a number of systematic questions which might blur the argument against EU. Corresponding remarks will apply to fits to the other three sets of data.

Firstly, could the disappearance of the predicted $f_{0}(980)$ arise from effects of the $K K$ threshold? The answer is no, because the $f_{0}$ appears clearly in elastic scattering and the prediction of EU is that it should be almost identical in production. A precise cancellation of the magnitude and phase of the predicted $f_{0}$ would be needed. This is ruled out by the known couplings of both $\sigma$ and $f_{0}$ to $K K$. The analytic continuation for the $f_{0}(980)$ below the $K K$ threshold is accurately determined over the small mass range concerned by BES data on $\phi \pi \pi$ and $\phi K K$. In the $\sigma$ amplitude, the $K K$ inelasticity rises over a mass scale of $200 \mathrm{MeV}$ as shown in Fig. 11 of [19]. Any flexibility in the analytic continuation of the $K K$ term then has a scale of $\sim 300 \mathrm{MeV}$. Furthermore, it is closely constrained by the fit to Kloe data on $\phi \rightarrow \gamma \pi^{0} \pi^{0}$. So this explanation is highly implausible.

Secondly, could the BES data be fitted assuming $J / \Psi \rightarrow \omega K K$, followed by $K K \rightarrow \pi \pi$ ? This has been tested by adding $T_{12}$ fitted freely. Its magnitude optimises at zero within errors. It improves log likelihood only by 2 .

\subsection{An objection of Aitchison}

Thirdly, Aitchison argues [24] that a further term $C$ might be added to the $P$-vector due to dynamics of the production process. A similar approach was used in fits by Bowler et al. and Basdevant and Berger to the $a_{1}(1260)$ to allow for the Deck effect [25-27]. An additional term $C \mathrm{e}^{\mathrm{i}\left(\delta_{A}+\delta_{B}\right)}\left(\cos \delta_{A} \cos \delta_{B}-\sin \delta_{A} \sin \delta_{B}\right)$ appears in $F \rho$. If $C=\mathrm{i} \alpha_{A}$, the second term can cancel the term $\mathrm{i} T_{A} T_{B}$ of $\mathrm{EU}$, leaving $T_{A}$ and the first of the additional terms, which is close to 0 since $\delta_{A} \simeq 90^{\circ}$. This removes almost all the structure due to $f_{0}(980)$. However, this cancellation also leaves a term i exp i $\left(\delta_{A}+\delta_{B}\right) \cos \delta_{A} \cos \delta_{B}$ and when $\delta_{B}$ is small this becomes $\mathrm{i} \exp \mathrm{i} \delta_{A} \cos \delta_{A}$, which is much larger than the term $T_{A}$ itself if $C$ is a constant. This is ruled out by the data, so it becomes necessary to tailor the $s$-dependence of $C$ to reproduce the magnitude of the $\pi \pi$ amplitude.

However, this is not the end of the story. Phase information is also available. In [14] it is shown that interference between $\sigma$ and $b_{1}(1235)$ measures the phase of the $\sigma$ amplitude (plus any background term $C$ ) and requires it to be the same as for elastic scattering down to $450 \mathrm{MeV}$ with an error of $5^{\circ}$ in the worst scenario, see Fig. 2 of that paper. It is worth mentioning that there is a similar result for the $\kappa$ in [15]. Figure 4 a of that paper shows that the $\kappa$ phase from BES data on $J / \Psi \rightarrow K^{+} \pi^{-} K^{-} \pi^{+}$agrees with the LASS effective range formula for elastic scattering down to $750 \mathrm{MeV}$ within $3.5^{\circ}$. Figure $4 \mathrm{f}$ of the same paper shows a corresponding agreement for E791 data on 
$D \rightarrow K \pi \pi$ within a similar error. These results rule out any background different from $\sigma$ and $\kappa$ with a magnitude larger than $12 \%$ and a phase difference above $\pm 5^{\circ}$. They of course agree with Watson's original statement that $D(s)$ of a single resonance should be universal.

There is no obvious source of the background proposed by Aitchison, as there was in [25], where a Deck background was visible in the data and made a $40 \%$ contribution to cancelling the $a_{1}(1260)$ amplitude. A similar fortuitous cancellation with unidentified backgrounds will be required also for all three of the following sets of data. In all cases, the additional background gives rise to a phase variation different to strict EU. If the background removes the $f_{0}$, only the $\sigma$ is left, i.e. $T_{\sigma}$, which contributes only part of the phase of elastic scattering; so the amplitude does not satisfy EU.

\section{$3.3 \bar{p} p \rightarrow 3 \pi^{0}$}

A fresh analysis of Crystal Barrel data on $\bar{p} p$ annihilation at rest to $3 \pi^{0}$ has been completed recently [5]. Data are available with statistics of $\sim 700 \mathrm{~K}$ events (and $0.5 \%$ experimental backgrounds) in both liquid and gaseous hydrogen; these allow a good separation of ${ }^{1} S_{0}$ and ${ }^{3} P_{1}$ initial states. Although the $f_{0}(980)$ appears clearly in ${ }^{1} S_{0}$ annihilation, the magnitude of the $\pi \pi S$-wave amplitude does not go to zero on the Argand diagram near $1 \mathrm{GeV}$, as EU predicts. This Argand diagram is reproduced in Fig. 6a. Its magnitude is smallest at $0.98 \mathrm{GeV}$, but is still very distinct from zero. For ${ }^{3} P_{1}$ annihilation, the fitted $f_{0}(980)$ amplitude, relative to $\sigma$, is much smaller, see Fig. 6b. The magnitude of the $\sigma$ amplitude is quite large near $1 \mathrm{GeV}$.

The question arises how robust these solutions are. Could they 'bend' to accomodate EU? The published analysis requires some $s$-dependence of the numerator fitted to the $\sigma$ amplitudes, of the form $(1+\Lambda s)$, with complex
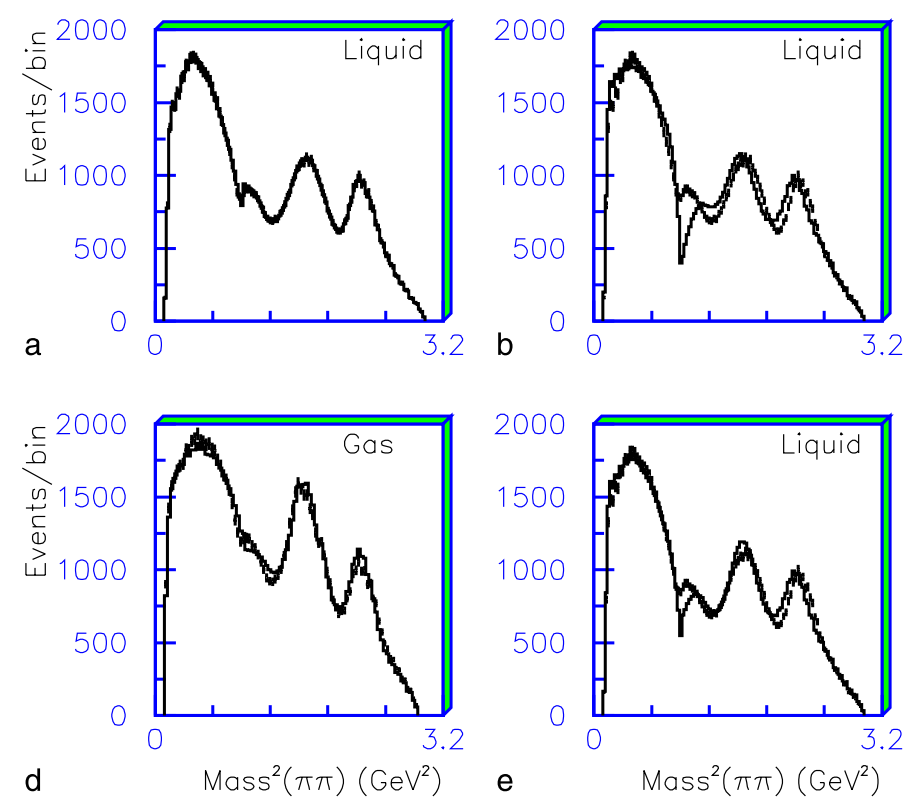
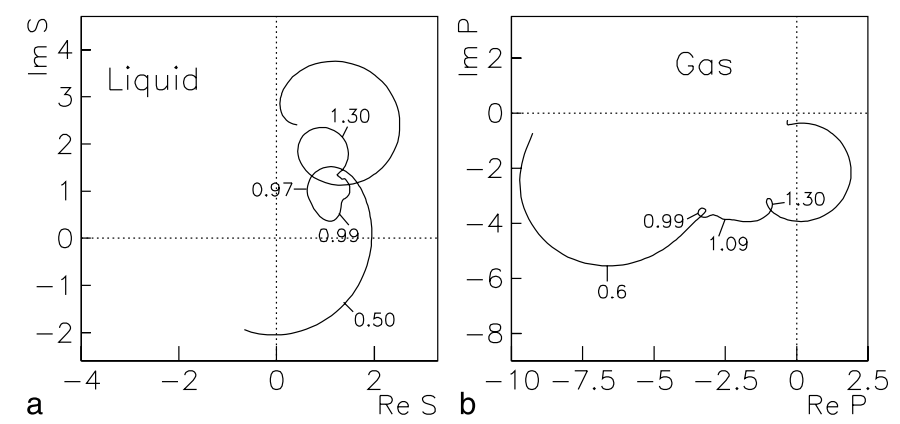

Fig. 6. Argand diagrams for the $\pi \pi S$-wave of [5] for $\mathbf{a}^{1} S_{0}$ annihilation, $\mathbf{b}{ }^{3} P_{1}$

A. Can extra flexibility reach agreement with EU? A second point is that the analysis does not include the repulsive $I=2 \pi \pi S$-wave. Could this bring conclusions into line with EU?

The brief answer is definitely no, and will be presented graphically. Fig. 7a shows the $\pi \pi$ mass projection for data in liquid from the current analysis; it fits the data points accurately. In Fig. 7b, the $\sigma+f_{0}$ combination of the isobar model is replaced by the EU combination (with the constraint from analyticity that magnitudes of $\sigma$ and $f_{0}(980)$ should be equal within $12 \%$ ). Initially, only the coupling constant of this combination is refitted, leaving other amplitudes untouched; this is for the purpose of illustrating the change required by EU. The $\sigma$ component is large and cannot change much; a deep dip appears at $1 \mathrm{GeV}$ because of the corresponding dip in the elastic amplitude.

It is of course necessary to re-optimise all components. The resulting mass projection is shown in Fig. $7 \mathrm{c}$ and is still in severe disagreement with the data. A measure of the disagreement may be obtained from $\chi^{2}$. Here, it is necessary to point out that even the fit of Fig. 7a has a $\chi^{2}$ larger than 1 . This is probably because of the enormous statistics and small, slowly varying systematic errors in acceptance. The
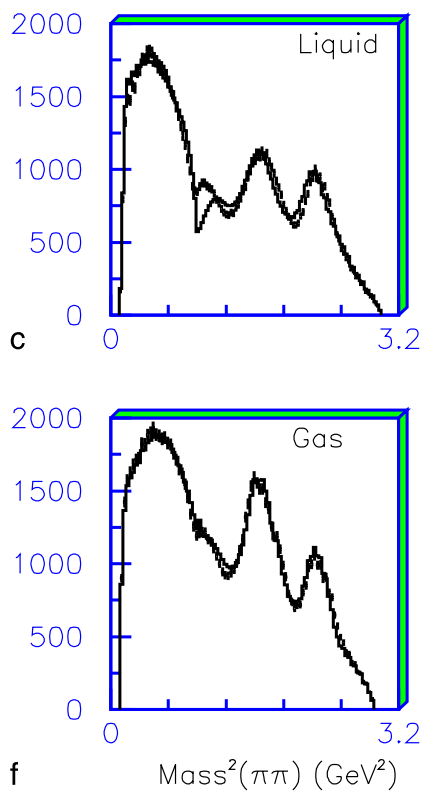

Fig. 7. $\pi \pi$ mass projection of data and fits: a from [5] in liquid hydrogen, $\mathbf{b}$ with the fitted EU amplitude but other amplitudes untouched, $\mathbf{c}$ after refitting all amplitudes, $\mathbf{d}$ as $\mathbf{c}$ in gaseous hydrogen, $\mathbf{e , f}$ with the $I=2 S$-wave included in the fit 
procedure adopted here is to renormalise $\chi^{2}$ to 1 per point for this fit and apply the same scaling factor to all other fits. The fit of Fig. 7c then has a renormalised $\chi^{2}$ of 40599 for 3500 bins; this is a 170 standard deviation discrepancy, allowing for the reduction in the number of fitted parameters by 2 .

A much better fit is possible if relative magnitudes of $\sigma$ and $f_{0}(980)$ are allowed to vary. The phase of the $S$ wave amplitude in Fig. 6 a is close to EU, and only a $10 \mathrm{MeV}$ shift is required for a perfect fit. This is consistent with the energy calibration and resolution of the Crystal Barrel detector. However, the fitted combination of amplitudes no longer agrees with the crucial equation (14).

Figure 7 f shows the effect of including the $I=2 S$-wave amplitude, using the formulae of Sect. 4.1 of [5]. There is only a rather small improvement, because the slow $s$ dependence of this amplitude cannot fill the narrow dip at $1 \mathrm{GeV}$. The renormalised $\chi^{2}$ falls to 34345 , a discrepancy of $\sim 130 \sigma$.

It is not just the $\pi \pi$ mass projection which governs $\chi^{2}$. One should inspect discrepancies in $\chi^{2}$ all over the Dalitz plot. Figure 8 makes such a comparison. Panel a shows discrepancies in $\chi^{2}$ where the fit is above the liquid data and panel $\mathrm{b}$ the discrepancies where the fit is low. Panels c and d show results for data in gas. One sees striking systematic discrepancies all over the Dalitz plot, arising from interferences. Such discrepancies are almost completely absent from the fit of Fig. 7a using the isobar model.

There are two further points for discussion. In the work shown on Fig. 7e and f, the $I=2 S$-wave was fitted without factoring out the Adler zero which occurs at $s \sim-0.41 m_{\pi}^{2}$. If this is done (as for the $\sigma$ amplitude), the broad $I=2$ amplitude gets confused with the $\sigma$ amplitude and leads to minor improvements all over the Dalitz plot. However, none of these is distinctive enough to require the $I=2 \mathrm{am}$ plitude definitively.

Secondly, could a more complicated polynomial than $(1+\Lambda s)$ multiply the $\sigma$ amplitude and give a successful fit? Extensive tests were made in [5] with the objective of improving the fits reported there. If one chooses too free a polynomial, the fitted ${ }^{3} P_{1}$ component in gaseous hydrogen can fluctuate wildly from the $50 \%$ predicted from Stark mixing by Reifenrofer and Klempt [28]. To avoid this, the fitted ${ }^{3} P_{1}$ component is constrained within the range $(50 \pm 7) \%$. Unless the numerator of the EU amplitude is designed to include a narrow dip at $1 \mathrm{GeV}$, no large improvement is observed.

One further observation from the Crystal Barrel data is worth reporting. Relative intensities of $f_{2}(1565)$ and $f_{2}(1270)$ are quite different in $\bar{p} p$ annihilation to those in elastic scattering. In $\bar{p} p$ data, the integrated intensities of these two resonances are equal within $12 \%$ after allowing
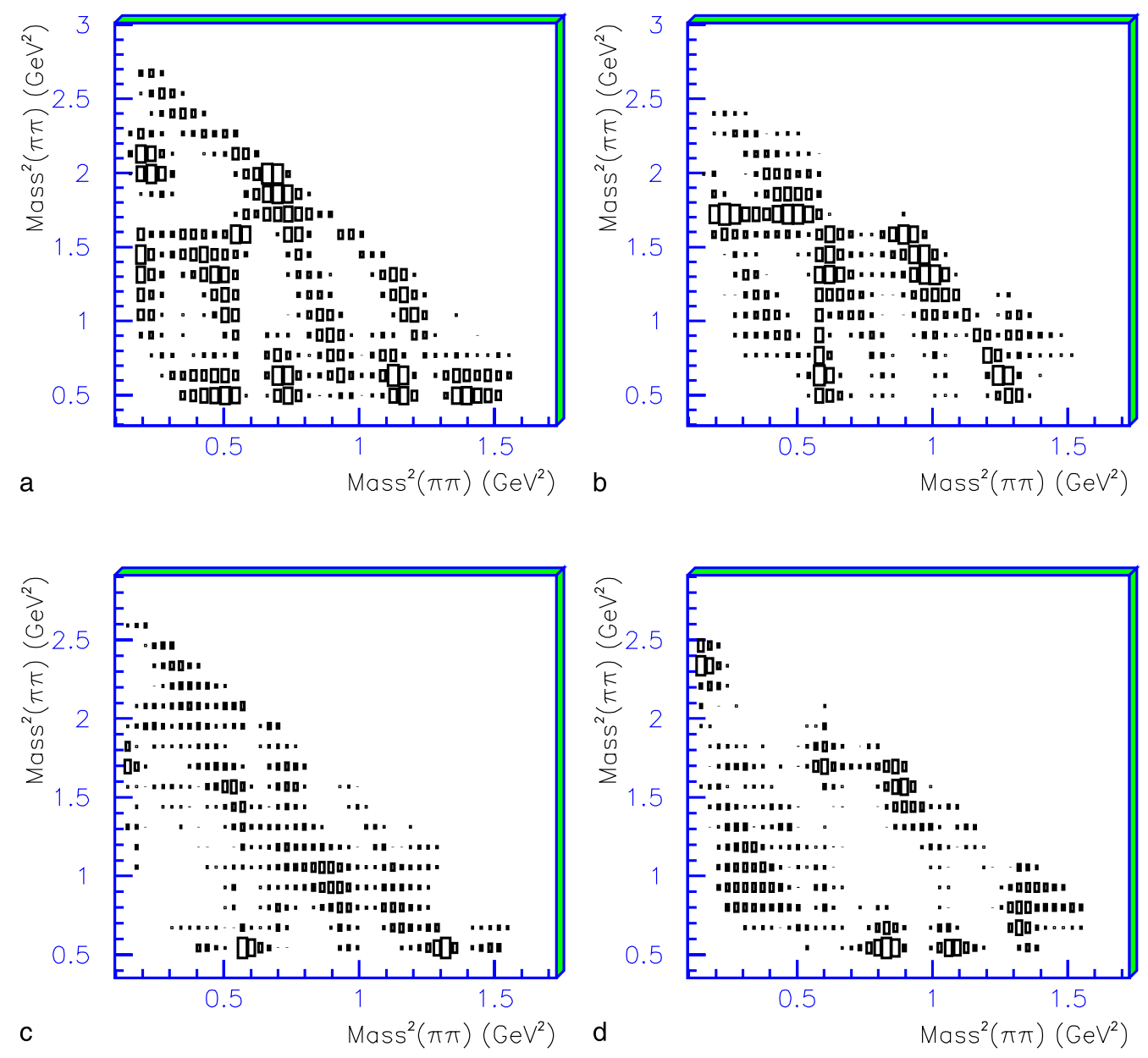

Fig. 8. Discrepancies in $\chi^{2}$ over the Dalitz plot for $\mathbf{a}, \mathbf{b}$ liquid hydrogen, c,d gaseous hydrogen. In $\mathbf{a}$ and $\mathbf{c}$ the fit is above the data and in $\mathbf{b}$ and $\mathbf{d}$ below 
for the (modest) effects of centrifugal barriers for production $\left(L=2\right.$ for ${ }^{1} S_{0}$ annihilation and $L=1$ for $\left.{ }^{3} P_{1}\right)$. However, in elastic scattering the fitted $f_{2}(1565) \rightarrow \pi \pi$ width is a factor 4 smaller than that of $f_{2}(1270) \rightarrow \pi \pi$. This again disagrees with EU.

\section{Central production of $\pi \pi$}

Central production of a $\pi \pi$ pair in $p p \rightarrow p p(\pi \pi)$ was fitted using EU by Au, Morgan and Pennington (AMP) [12]. In data from the AFS experiment at the ISR [10], the two final-state protons are produced with very small 4-momentum transfers $t=-0.003 \mathrm{GeV}^{2}$. It is routinely assumed that the $\pi \pi$ pair is unaffected by final state interactions with these protons, which are separated from the central region by a gap in rapidity.

AMP draw attention to structure in the $\pi \pi$ mass spectum similar to the dip in the $\pi \pi S$-wave elastic cross section. Figure 9 shows the AFS data as open squares. There is a peak at low $\pi \pi$ mass, close to that of the $\sigma$ pole in BES data, but not quite identical, for reasons discussed shortly.

Triangles on Fig. 9 show $\pi \pi$ elastic cross sections derived from Cern-Munich phase shifts; this is done by dividing the $\pi \pi$ amplitude of elastic scattering by $N(s)$, leaving only the term $1 / D(s)$. The curve shows my fit to elas- tic data after dividing the $\pi \pi$ amplitude by $N(\mathrm{el})$. The agreement between the curve and triangles demonstrates that the parametrisation of the $\sigma$ reproduces Cern-Munich phase shifts. Results are similar to the AFS data, but there is a distinct difference in the vicinity of $f_{0}(980)$. What is clearly evident is constructive interference between $f_{0}$ and $\sigma$ immediately below $989 \mathrm{MeV}$, where EU predicts a zero. Even without fitting, one can see that EU will fail.

A detail is that, up to the $K K$ threshold, one piece of information from each Cern-Munich moment is sufficient to determine phase shifts for both $S$ - and $P$-waves. Above the $K K$ threshold, however, the separation of inelasticity parameters $\eta$ and phase shifts $\delta$ cannot be made without further assumptions. Just above the $K K$ threshold, the solution of Ochs [16] has $\eta=0.6-0.7$, whereas the BES lineshape for $f_{0}(980)$ demands $\eta$ parameters dropping to $\sim 0.2$ at $1.01 \mathrm{GeV}$. In view of this large discrepancy, predictions from Cern-Munich phase shifts are not shown above the $K K$ threshold.

Since the work of Morgan and Pennington [29], information on both $\sigma$ and $f_{0}(980)$ has improved enormously. The $\sigma$ amplitude is taken (within errors) from [13], using fit (iii) given there. The resulting cross section is shown by the chain curve of Fig. 10a. Although this fit follows the general features of the data, it is not accurate for low $\pi \pi$ masses. Varying parameters of $f_{0}(980)$ within errors quoted by BES has negligible effect on the quality of the fit.

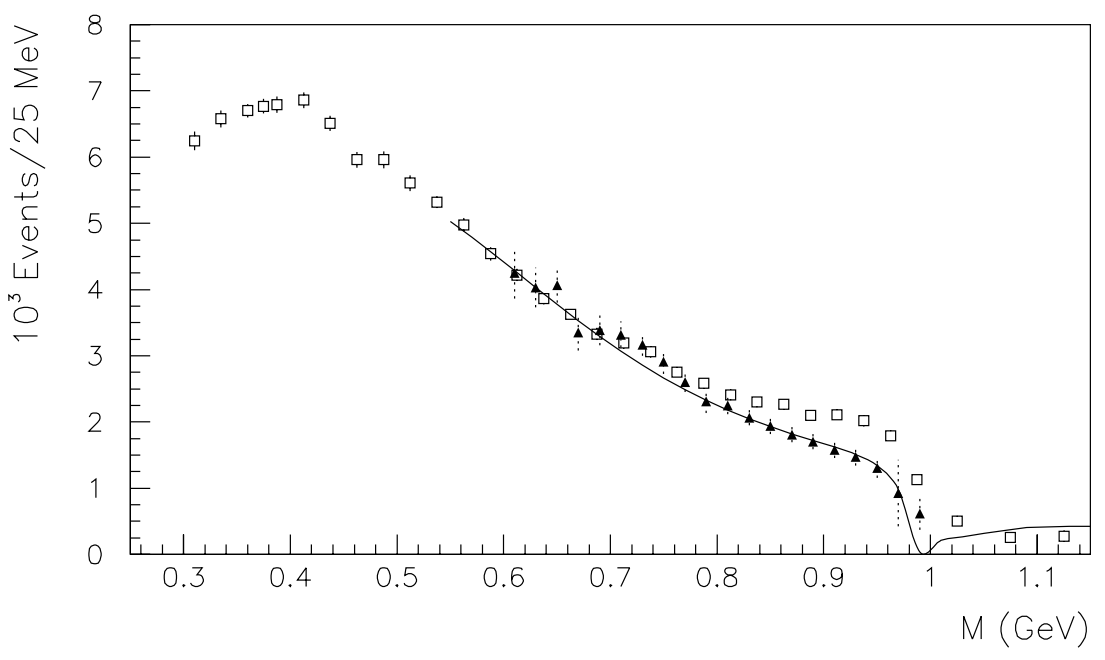

Fig. 9. Open squares, AFS data; triangles, $\pi \pi$ cross sections derived from phase shifts of Ochs [16]; curve, the elastic cross section with the numerator $N(s)$ replaced by a constant

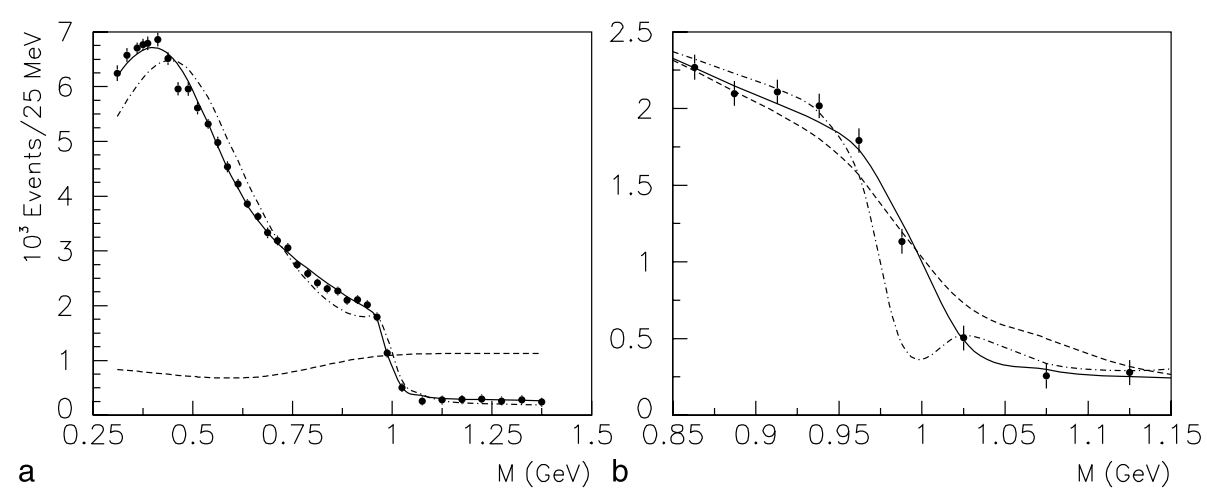

Fig. 10. Fits to AFS data: a full curve isobar model, chain curve using the $\sigma$ amplitude of [15], dashed curve the scaling factor for the final fit. $\mathbf{b}$ enlargement of the $f_{0}(980)$ region: full curve isobar model, chain curve EU fit, dotted $\mathrm{EU}+$ freely fitted $I=2 S$-wave 
Some additional flexibility is clearly needed in the numerator $X(s)$ of the $\sigma$ amplitude.

There are two obvious sources. Firstly, AMP point out that central production goes via two intermediate Pomerons: $P P \rightarrow \pi \pi$, where $\pi$ exchange is allowed. This alone does not achieve a good fit. The second explanation arises from Regge factors in the production process. AMP argue for an $s$-dependent factor $\left(m_{\rho}^{2}+s\right)$ in the numerator arising from the leading Regge trajectory. There may be contributions also from a daughter trajectory.

The full curve of Fig. 10a shows an isobar model fit using a complex constant for $f_{0}(980)$, but without any $f_{0}(1370)$. It uses a scaling factor for the cross section shown by the dashed curve. This takes the form of a Gaussian dip: $N(\operatorname{prodn})=1-A \exp -\left(s-s_{0}\right)^{2}$. This scaling factor gives rise to a slow modulation of the $\sigma$ amplitude over hundreds of $\mathrm{MeV}$. It has only a small effect on what is fitted to $f_{0}(980)$. A detail is that a Gaussian mass resolution of $10 \mathrm{MeV}$, quoted by the AFS collaboration [10], is folded into each point of the fit; it is significant only near the $K K$ threshold. The fit to $\pi \pi$ data has a $\chi^{2}$ of 35.2 for 34 degrees of freedom.

Consider now the $f_{0}(980)$ mass range. The isobar model fit of Fig. 10a requires an $f_{0}$ magnitude only $\sim 60 \%$ of the EU prediction. This fit requires constructive interference between $\sigma$ and $f_{0}$ below the $f_{0}$ mass and destructive interference above. It implies the $f_{0}$ phase is $(57 \pm 7)^{\circ}$ below the EU prediction. This immediately throws doubt on EU.

A fit using EU is conspicuously bad. As an example, if the first 29 points up to $988 \mathrm{MeV}$ are fitted alone, $\chi^{2}=$ 141.7 if fitted by $\sigma$ and $f_{0}(980)$ only. EU predicts an amplitude which is almost zero at $989 \mathrm{MeV}$, whereas the data at $988 \mathrm{MeV}$ are far above this. If the EU fit is extended to $1300 \mathrm{MeV}$ including the $f_{0}(1370)$ with the elasticity fitted in [5], $\chi^{2}$ is $>10^{4}$, because the $f_{0}(1370)$ contribution is far too large. Even if the $f_{0}(1370)$ is fitted freely in magnitude, $\chi^{2}=137$ for 37 points. The fit, shown by the chain curve of Fig. 10b is particularly bad close to the $K K$ threshold, where the dip of elastic scattering is predicted by EU. The fit to $K K$ data is also poor, with a $\chi^{2}$ of 18 for 5 points.

The dotted curve of Fig. 10b shows the effect of fitting freely an additional contribution from the $I=2 S$-waves: $\chi^{2}=59.6$. This fails to cure the poor fit. It makes almost no difference whether the $I=2$ amplitude is divided by a factor $\left(s-s_{A}\right)$ like the $\sigma$ amplitude. The basic difficulty is that the slowly varying $I=2$ amplitude cannot cure the rapid structure due to $f_{0}(980)$. Furthermore, the relative magnitudes of the fitted $I=2$ amplitude and the EU amplitude is 0.46 , whereas it is only 0.18 for elastic scattering at $1 \mathrm{GeV}$. Such a large $I=2$ amplitude is implausible.

Obviously the problem with EU is that the magnitude of the $f_{0}$ amplitude needs to be smaller than for elastic scattering. From analyticity, this also requires a difference in phase. That is also clear from the absence of the predicted dip at $989 \mathrm{MeV}$ in the data.

If the phase of the $f_{0}(980)$ amplitude is constrained to the EU value, but relative magnitudes of $\sigma$ and $f_{0}$ are set free, $\chi^{2}=144$, which is bad. Most of $\chi^{2}$ comes from points immediately around the $K K$ threshold, showing that the data reject also the phase variation of EU.

\subsection{Proposed modification to EU}

At this point, one could argue that the mechanism of the production reaction is unknown and might generate a phase for $f_{0}(980)$ different to that of the $\sigma$. This argument is not specific, though the isobar model can fit the data well. However, the usual argument for a different phase for $f_{0}$ and $\sigma$ is multiple scattering of the pions with spectator particles. In AFS data, there is an empty rapidity gap isolating the central region. Remember also that CernMunich phase shifts are derived in the first instance from data on $\pi p \rightarrow \pi \pi p$ at high momentum and small momentum transfer, a similar configuration.

The conjecture of EU will now be replaced with an alternative ansatz. The treatment of production data needs to be able to cope with the case where one resonance amplitude is zero. EU does not, since a universal phase equal to elastic scattering requires a production amplitude $T^{(p)} \propto 1 / D^{\sigma}(s) D_{0}^{f}(s)$. The correct production amplitude should reduce to $T^{\sigma}$ when the $f_{0}$ is absent. A small $f_{0}$ amplitude should produce a small perturbation to $T_{\sigma}$.

Suppose the 2-body $\pi \pi$ amplitude is written as

$$
F \rho_{1}=\alpha T^{(p)}=\frac{\alpha^{\prime}}{\sqrt{1+\beta^{2}}}\left[T_{A}+\beta T_{B} \mathrm{e}^{2 \mathrm{i} \Psi(s)}\right],
$$

where $\alpha^{\prime}$ and $\beta$ are real. This allows freedom in $\beta$ and includes a phase $\Psi(s)$ which becomes the same as for elastic scattering when $\beta \rightarrow 1$. It is necessary to choose as $A$ the state with the larger amplitude on resonance, so that $\beta \leq 1$. If one could create this $\pi \pi$ system in 'free space', the appropriate 2-body unitarity relation below the $K K$ threshold would be

$$
\operatorname{Im} T^{(p)}=\left|T^{(p)}\right|^{2} .
$$

An alternative way of formulating the basic physics (with the same outcome) is in terms of the SchwingerDyson equation. Instead of the conventional relation

$$
T_{\text {prod }}=V_{\text {prod }}+V_{\text {prod }} G T_{\text {el }},
$$

my conjecture is to replace this with

$$
T_{\text {prod }}=V_{\text {prod }}+V_{\text {prod }} G T_{\text {prod }} .
$$

Here $V$ is the 'potential' generating the final state and $G$ is the propagator.

For the case of purely elastic scattering, a closed form for $\Psi(s)$ of (19) may be derived by substituting $T_{A}$ and $T_{B}$ in the form $\left(\mathrm{e}^{2 \mathrm{i} \delta}-1\right) / 2 \mathrm{i}$ into (20). After simple cancellations between left- and right-hand sides,

$$
\sin \left(2 \Psi+\delta_{B}-2 \delta_{A}\right)=\beta \sin \delta_{B}
$$

or

$$
2 \Psi=2 \delta_{A}-\delta_{B}+\sin ^{-1}\left(\beta \sin \delta_{B}\right) .
$$

If $\beta \neq 1$, this is a different relation from purely elastic scattering. 
The improvement in the fit is dramatic. Immediately an excellent fit to points below the $K K$ threshold is obtained with $\chi^{2}=28.4$ for 29 points and 24 degrees of freedom. The term $\sin ^{-1}\left(\beta \sin \delta_{B}\right)$ in (24) differs from $\delta_{B}$ by $54^{\circ}$. This is just what is needed to produce the interference between $\sigma$ and $f_{0}$ observed in the isobar model fit.

Above the $K K$ threshold, it is tempting to satisfy unitarity by introducing a $K$-matrix. However, the $K$-matrix depends on the assumption that the 2-body system is confined to the unitary circle, but that is no longer the case in a 3-body situation.

The fit may be extended above the inelastic threshold by writing $\pi \pi$ and $K K$ amplitudes as

$$
\begin{aligned}
F_{\pi \pi} & =\alpha\left[T_{11}^{\sigma}+\gamma T_{21}^{\sigma}+\left(\beta T_{11}^{f}+\epsilon T_{21}^{f}\right) \mathrm{e}^{2 \mathrm{i} \Psi}\right] / \rho_{1} \\
& =\alpha\left[T_{11}^{\sigma}\left(1+\frac{\gamma g_{2}^{\sigma} r}{g_{1}^{\sigma}}\right)+T_{11}^{f}\left(\beta+\frac{\epsilon g_{2}^{f} r}{g_{1}^{f}}\right) \mathrm{e}^{2 \mathrm{i} \Psi}\right] / \rho_{1}, \\
F_{K K} & =\alpha\left[T_{12}^{\sigma}+\gamma T_{22}^{\sigma}+\left(\beta T_{12}^{f}+\epsilon T_{22}^{f}\right) \mathrm{e}^{2 \mathrm{i} \Psi^{\prime}}\right] / \rho_{2} \\
& =\alpha\left[T_{11}^{\sigma} \frac{g_{2}^{\sigma}}{g_{1}^{\sigma}}\left(\frac{1}{r}+\frac{\gamma g_{2}^{\sigma}}{g_{1}^{\sigma}}\right)+T_{11}^{f} \frac{g_{2}^{f}}{g_{1}^{f}}\left(\beta+\frac{\epsilon g_{2}^{f}}{g_{1}^{f}}\right) \mathrm{e}^{2 \mathrm{i} \Psi^{\prime}}\right] / \rho_{1} .
\end{aligned}
$$

Equations (25) and (26) expose the explicit dependence of $T_{12}$ on the ratio $r=\sqrt{\rho_{2} / \rho_{1}}$; the experimental group divides out the phase space $\rho_{2}$ and $\rho_{1}$ in the $\pi \pi$ and $K K$ channels. As explained in Sect. 2.1, all $T$ have the numerator of elastic scattering replaced by a constant.

My $T_{12}$ is defined so as to contain a factor $\sqrt{\rho_{1} \rho_{2}}$ and $T_{22}$ is defined to contain a factor $\rho_{2}$. With these definitions, the unitarity relations become

$$
\begin{aligned}
& \operatorname{Im} T_{11}=\left|T_{11}\right|^{2}+\left|T_{12}\right|^{2}, \\
& \operatorname{Im} T_{12}=T_{11}^{*} T_{12}+T_{12}^{*} T_{22}, \\
& \operatorname{Im} T_{22}=\left|T_{22}^{*}\right|^{2}+T_{21}^{*} T_{12} .
\end{aligned}
$$

Equations (25) and (26) satisfy these relations by construction, except that $\Psi$ and $\Psi^{\prime}$ need to be constrained to obey (27)-(29). Above the $K K$ threshold, this is done using freely fitted $\Psi$ and $\Psi^{\prime}$ for every data point and introducing into $\chi^{2}$ a penalty function which applies (27)-(29) with $3 \%$ errors; in practise these constraints are easily satisfied and discrepancies at the end of the fit are below the $1 \%$ level; this is well below experimental errors. In fact the $K K$ data are not very precise, leaving large flexibility in $\Psi^{\prime}$ above the $K K$ threshold. In other words, the data are easy to fit above the $K K$ threshold, but highly definitive below it.

A detail is that $g_{2}$ needs to include form factors both below and above the $K K$ threshold, such that it falls quite rapidly on both sides of the threshold; formulae are given in [13].

The best fit with $\sigma$ and $f_{0}(980)$ alone has $\chi^{2}=46.4$ for 37 points and 32 degrees of freedom. The fit is good up to $1.1 \mathrm{GeV}$, but is inadequate for $\pi \pi$ data near $1.3 \mathrm{GeV}$. This may be cured straightforwardly by adding a small $f_{0}(1370)$ component. A technical detail is that the $f_{0}(1370)$ amplitude is multiplied by a factor $\exp \left(2 \mathrm{i} \Psi^{\prime \prime}\right)$ and (24) is iterated; the contribution of $f_{0}(1370)$ to $T_{12}$ and $T_{22}$ is negligible. The resulting fit, shown on Fig. 11a, has $\beta=0.59 \pm$ 0.06 and the $f_{0}(1370)$ amplitude is 0.18 times that of the $\sigma$ amplitude at $1.3 \mathrm{GeV}$. The $\chi^{2}$ for $\pi \pi$ data is 28.7 for 29 degrees of freedom. The Omega collaboration reports a significant contribution from $f_{0}(1370)$ to their data on central production of $\pi^{+} \pi^{-}$[30]. Their fitted mass and width agree closely with the line-shape fitted to $f_{0}(1370)$ in [5].

Figure 11b shows the fit to AFS $K^{+} K^{-}$data. A detail here is that the $K^{+} K^{-}$data of AFS are scaled up by a factor $4 / 3$ to allow for isospin Clebsch-Gordan coefficients in $\pi^{+} \pi^{-}$and $K^{+} K^{-}$systems. The $K K$ data constrain the coefficients of $T_{12}$ and $T_{22}$ amplitudes. The lowest AFS $K K$ point has small acceptance which may have significant systematic uncertainty [31].

Figure 12 shows the Argand diagram for the fitted amplitude. This illustrates the form of (20). There is a geometrical relation between the imaginary part of the amplitude and its modulus squared, but it is a different relation to EU. The $f_{0}(980)$ amplitude is smaller than that of the $\sigma$ and their relative phases are different to EU. The same is
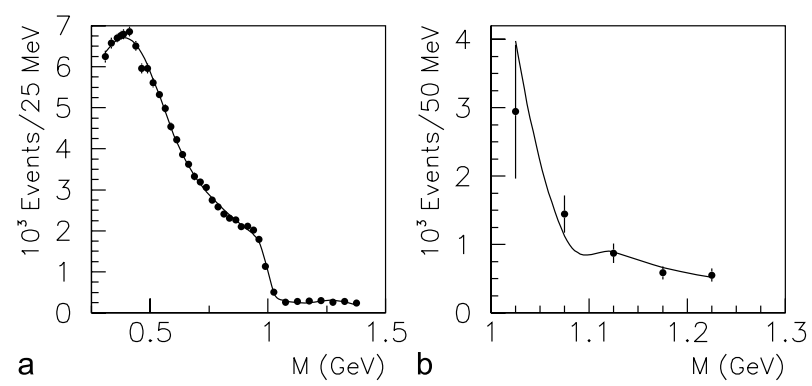

Fig. 11. Fits to AFS data: a using the revised form of 2-body unitarity, b fit to $K K$ data

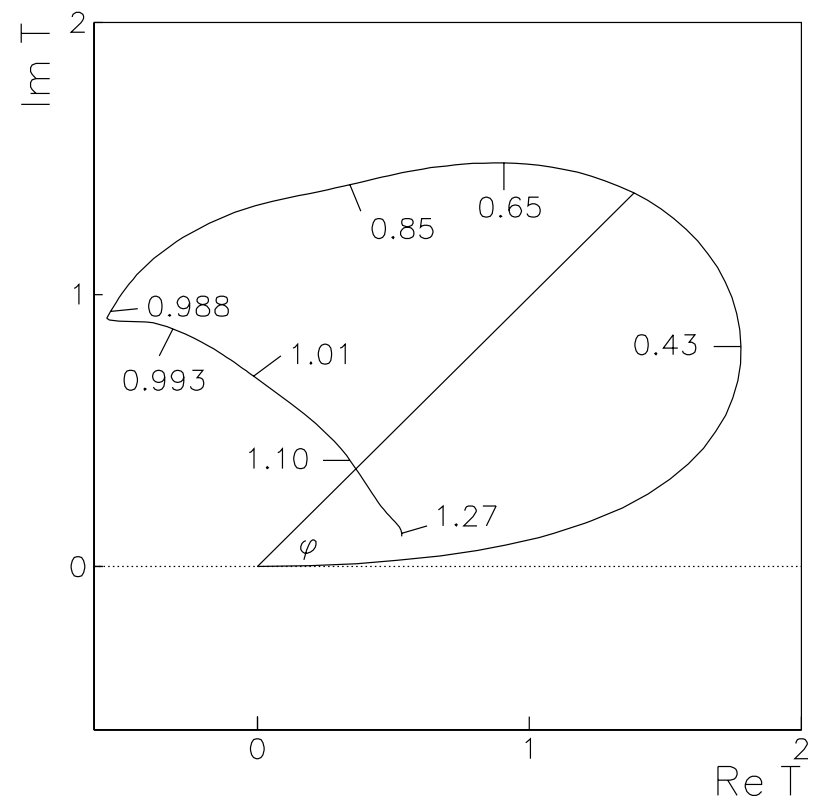

Fig. 12. The Argand diagram of the amplitude fitting AFS data; masses are marked in $\mathrm{GeV}$ 
true of Fig. 5, the Argand diagram fitting BES data; there the $f_{0}(980)$ contribution is very small.

Let us now return to (20) and (24) and review their general features. The last term of $(24), \sin ^{-1}\left(\beta \sin \delta_{B}\right) \rightarrow \delta_{B}$ as $\beta \rightarrow 1$ and $\delta_{B} \rightarrow 90^{\circ}$. Furthermore it approaches this limit non-linearly as $\beta \rightarrow 1$. The interesting point is that two-body elastic scattering emerges as a limiting case of a more general relation. Furthermore it has pathological properties as the relative magnitude of $A$ and $B$ crosses 1 (or -1). If $B$ becomes larger than $A$, it is necessary to interchange the roles of $A$ and $B$. As $\beta$ approaches 1 from below, $\Psi \rightarrow \delta_{A}$ and one recovers the standard result of elastic scattering. Further pathological cases arise if $\beta \rightarrow-1$ (impossible in the 2-body elastic case). Elastic scattering is in fact a very special case. So EU can fail very badly as $\beta$ departs from 1.

As $\beta$ drops from 1, the phase $\Psi$ measured from the origin of the Argand diagram soon changes by only a modest amount over the $f_{0}$. In this case, the isobar model becomes an excellent approximation: the $f_{0}$ has its usual dependence on $s$ through its phase shift $\delta$ and the $f_{0}$ amplitude adds vectorially to the $\sigma$; the isobar model can then fit a constant phase to both quite successfully if the term $\sin ^{-1}(\beta \sin \delta)$ is small.

In summary, the modified form of EU suggested in (20) and (24) gives a much better fit than EU. The fit of Morgan and Pennington [29] using EU requires an additional thirdsheet pole at $M=978-\mathrm{i} 28 \mathrm{MeV}$. This additional pole cannot be accomodated by BES $\phi \pi \pi$ data, which require only a second-sheet pole at $998-\mathrm{i} 17 \mathrm{MeV}$ and a broad thirdsheet pole at $851-\mathrm{i} 418 \mathrm{MeV}$. These two poles have a natural explanation. If the coupling of $f_{0}(980)$ to $K K$ is allowed to decrease gradually to zero, leaving other parameters unchanged, the two poles coalesce towards the same pole position $M=968-\mathrm{i} 82 \mathrm{MeV}$; it is the effect of $\rho(K K)$ which moves the second-sheet pole to the $K K$ threshold and moves the third-sheet pole away. There is no pole in this mass range from the $\sigma$ amplitude.

A second remark is that all of $\sigma, \kappa, a_{0}(980)$ and $f_{0}(980)$ may be reproduced as a nonet by the model of Rupp and van Beveren, where mesons couple to a quark loop [32]. In this model, no additional pole like that of Morgan and Pennington appears in the mass range close to the $K K$ threshold. These two results suggest that the additional pole is a consequence of the constraint of $\mathrm{EU}$.

The relation (20) is a new conjecture. Are there forseeable snags? The $\sigma$ and $f_{0}$ may mix, and this mixing could be different in elastic scattering and production. This mixing would alter the apparent width of $f_{0}(980)$ and could induce an additional phase change relative to $\sigma$. Presently there is no indication of any need for mixing. Such mixing will be absent if $\sigma$ and $f_{0}(980)$ have strictly orthogonal wave functions, as is plausible for members of the same nonet. On Fig. 2, the $\sigma$ is definitely visible in $\phi \pi \pi$ data. It would not be surprising if $\phi \pi \pi$ and $\omega \pi \pi$ channels filter out orthogonal combinations of $\sigma$ and $f_{0}$. From Fig. 2, one can estimate the relative intensities of $\sigma$ and $f_{0}$. It is necessary to allow for the mass resolution, since the $f_{0}$ amplitude falls extremely rapidly from its peak at $989 \mathrm{MeV}$, particularly above the $K K$ threshold. Doing this, the in- tensity of $\sigma$ is $4 \%$ of $f_{0}$ at the peak, i.e. $20 \%$ in amplitde. This is marginally higher than the $f_{0}$ signal fitted to $\omega \pi \pi$ but within the error, supporting the idea of orthogonal amplitudes.

\section{How to fit elastic data above the $K K$ threshold}

Many authors use the $K$-matrix to satisfy unitarity for 2-body scattering, e.g. the coupled channels $\pi \pi, K K, \eta \eta$, $\eta \eta^{\prime}$ and $4 \pi$. The popular approach is to add $K$-matrices of all resonances appearing in one partial wave. However, if resonances overlap, as $\sigma$ and $f_{0}(980)$ do, the $K$-matrix poles occur at masses where combined phase shifts happen to go through $90^{\circ}, 270^{\circ}$, etc., i.e. at $\sim 750$ and $1200 \mathrm{MeV}$. Firstly, an expansion in terms of these poles is problematical for $f_{0}(980)$ unless other factors or high powers of $s$ are included. Secondly, the relation between $K$-matrix poles and $T$-matrix poles is obscure. Any one $T$-matrix pole is built up from all $K$-matrix poles; the converse is obvious. The prescription that $S$-matrices multiply below the inelastic threshold does not appear naturally, but has to be enforced by fitting data.

An attractive alternative can be constructed following the spirit of Aitchison's approach (for 2-body scattering). Suppose one combines two resonances according to the prescription

$$
K_{i j}(\text { total })=\frac{\left(K_{A}+K_{B}\right)_{i j}}{1-0.5 \rho_{i} \rho_{j}\left(K_{A} K_{B}+K_{B} K_{A}\right)_{i j}} .
$$

Below the $K K$ threshold, this automatically gives the result that phase shifts add. (Further resonances may be combined by iterating this prescription). A nice feature of (30) is that one can write

$$
K_{i j}=\frac{g_{i} g_{j}}{M^{2}-s},
$$

using the same mass $M$ as the usual Breit-Wigner denominator. A second attractive feature of (31) is that the amplitude continues naturally through the $K K$ threshold, because of the factor $\rho_{i} \rho_{j}$ in the denominator.

My own approach in several papers, $[5,19]$ has been close to this. All diagonal elements of $S$-matrices are multiplied, as proposed here. Magnitudes of off-diagonal elements of the $S$-matrix need to be calculated from unitarity relations. For example, for a 3-channel system:

$$
\left|S_{12}\right|^{2}=(1 / 2)\left(1+\left|S_{33}\right|^{2}-\left|S_{11}\right|^{2}-\left|S_{22}\right|^{2}\right) .
$$

The phase of these off-diagonal elements has been fitted empirically, whereas (30) would predict these phases. This approach successfully fits elastic data, $\pi \pi \rightarrow K K$ and $\eta \eta$ with one proviso: a good fit requires inclusion of mixing between $\sigma, f_{0}(1370)$ and $f_{0}(1500)$ [5], using the formulae of Anisovich, Anisovich and Sarantsev [33]; these formulae are the modern equivalent of the Breit-Rabi equation of molecular spectroscopy, generalised to include resonance widths. 


\section{Conclusions}

Crystal Barrel data have $f_{0}(980)$ and $\sigma$ components with relative magnitudes seriously different to those predicted by EU plus analyticity. Furthermore, they are different in ${ }^{3} S_{1}$ and ${ }^{3} P_{1}$ annihilation. The BES data for $J / \Psi \rightarrow \omega \pi^{+} \pi^{-}$ do not reproduce the deep dip of elastic scattering at $989 \mathrm{MeV}$. AFS data likewise do not contain the same dip at this mass. All these results are in conflict with (14), which is a direct consequence of EU. This shows unambiguously that there must be a major flaw in the hypothesis of EU.

Experimentalists have dealt with this problem by using complex coupling constants for each resonance. However, as emphasised in Sect. 2.1, the imaginary part of the coupling constant introduces into the numerator of the amplitude an $s$-dependent phase variation which alters the universal phase coming from the denominator $[1-\mathrm{i} \rho K(s)]$. This destroys the original idea of a universal phase. One might as well fit directly in terms of the $T$-matrix of each individual resonance, along the lines outlined in Sect. 5. The form of the $K$-matrix suggested there would eliminate differences between $K$-matrix and $T$-matrix poles, making interpretation of results more direct.

Not all experimentalists adopt the $P$-vector approach. Some fit directly in terms of individual $T$-matrices for each resonance, including sequential decays from one resonance to a daughter with different complex coupling constants for each decay mode. Ascoli and Wyld [34] and Schult and Wyld [35] consider a multiple scattering series of the type $R \rightarrow(12) 3 \rightarrow 1(23)$, etc., where $R$ is a 3 -body resonance and brackets indicate resonances in two-body sub-systems; this is a unitarity effect of a different form to that considered here.

In view of the failure of $\mathrm{EU}$ in the 4 cases considered here, each new set of data should be inspected on its merits.

Let us examine ways of trying to save EU. Firstly, it is possible that unspecified backgrounds can be added to the P-vector so as to side-step the conflict. However, analyticity independently limits relative magnitudes of $f_{0}(980)$ and $\sigma$ within $12 \%$. Experimental determinations of $\sigma$ and $\kappa$ phases in [14] and [15] constrain phases within $\pm 5^{\circ}$. The probability that unspecified backgrounds can evade (14) to this accuracy in four sets of data is vanishingly small. Furthermore, if such backgrounds are introduced, EU loses any predictive power.

Secondly, Crystal Barrel data and AFS data cannot be fitted with EU whether or not the $I=2 S$-wave amplitude is included. So this is not a satisfactory escape route.

A third likely possibility, applicable to the first three sets of data, is that pions from sigma and/or $f_{0}(980)$ rescatter from the spectator particle, leading to a breakdown of EU. Aitchison himself pointed this out. Today, we known that such graphs have magnitudes typically $25 \%$ of those of the parent processes before the rescattering. This is sufficient to introduce large phase changes in some cases, but not all.

For all of these three sets of data, the isobar model provides an excellent fit. The production amplitude is then written $F=\alpha_{A} N_{A}(\mathrm{el}) / D_{A}+\alpha_{B} N_{B}(\mathrm{el}) / D_{B}$ with complex $\alpha_{A}$ and $\alpha_{B}$. In this form, no vestige remains of the con- straint that $S$-matrices must multiply as in elastic scattering. Relative magnitudes of $\alpha_{A}$ and $\alpha_{B}$ can arise from matrix elements coupling the initial state to each resonance. For $J / \Psi \rightarrow \omega \pi \pi$ and ${ }^{3} P_{1} \bar{p} p \rightarrow 3 \pi^{0}$, the $f_{0}$ component is so small that one cannot tell whether the phase alone follows EU or not. However, for ${ }^{1} S_{0} \bar{p} p \rightarrow 3 \pi^{0}$, the $f_{0}$ signal is large enough to rule out this possibility.

The fourth point is that one would still expect EU to work for AFS data, but it does not. An excellent fit may be obtained by replacing the unitarity relation $\operatorname{Im} T^{(p)}=$ $T^{(p)} T_{\mathrm{el}}^{*}$ by the new relation

$$
\operatorname{Im} T^{(p)}=\left|T^{(p)}\right|^{2} .
$$

This corresponds to the relation

$$
\operatorname{Im} F=F T^{*(p)},
$$

rather than the commonly used form $\operatorname{Im} F=F T_{\mathrm{el}}^{*}$.

Equations (20) and (24) have pathological behaviour in the vicinity of the 2-body elastic limit $\beta=1$. One can now see the basic problem of EU. It attempts to impose on the 3 -body system a very special behaviour which is narrowly restricted to 2-body scattering. Away from the special case $\beta=1$, the isobar model works successfully.

There are two points about the new unitarity relations. Firstly, it was argued in Sect. 2.1 that a universal phase in the denominator of the amplitude also requires, via analyticity, almost universal magnitudes; the word 'almost' covers the possibility that there may be slowly varying form factors or centrifugal barrier factors in production reactions without corresponding changes to $D(s)$. If relative magnitudes of resonances differ by large amounts between 2-body scattering and production, their relative phases must also change.

Secondly, the new unitarity relation (33) succeeds quantitatively in accounting for the observed relative phase between $\sigma$ and $f_{0}(980)$ in central production. That is a non-trivial result. The fit to AFS data then requires only two poles in the vicinity of $f_{0}(980)$, in agreement with the BES line-shape (as does the isobar model). EU requires an extra pole for which there is no obvious explanation. The form of this new unitarity relation is illustrated by the Argand diagrams of Figs. 5 and 12. In both, the $f_{0}(980)$ amplitude is small or fairly small and so is $\sin ^{-1}\left(\beta \sin \delta_{B}\right)$ of (19). As a result, the phase $\Psi$ measured from the origin of the Argand diagram changes rather little over the $f_{0}$. This is an extra source of phases appearing in the isobar model, and has not been appreciated before.

However, one then needs to ask whether this new relation can be used universally in the isobar model. Does it, for example, correctly describe the relative phases of $\sigma$ and $f_{0}$ in ${ }^{1} S_{0} \rightarrow 3 \pi^{0}$ data and in $J / \Psi \rightarrow \phi \pi \pi$ ? The answer is no. For these two reactions, the agreement between data and (20) and (24) improves substantially over EU. However, there are still discrepancies with the new unitarity relation of $20-30^{\circ}$, which is still significant. It seems likely that rescattering of pions from the spectator introduces some additional phases.

The new unitarity relation needs to be tested elsewhere. A possible testing ground is in Kloe data on $\phi \rightarrow \gamma \pi^{0} \pi^{0}$, 
where both $\sigma$ and $f_{0}$ may contribute, but the decay is electromagnetic; the small amplitude from $\phi \rightarrow \rho \pi^{0}$ introduces a perturbation of only $4 \%$ in amplitude and only in well defined parts of the Dalitz plot.

The remedy which succeeds well in fitting nearly all data is the isobar model, where both magnitudes and phases of resonances are both fitted freely. It needs to be emphasised that experimental groups have adopted the flexibility needed to fit existing data, so their results are essentially sound and are not in question. The hypothesis of EU has mostly been adopted by theorists for making predictions. Those predictions now need to be viewed with suspicion. Although the scalar form factor is well determined for elastic scattering, it is dangerous to assume that this form factor is universal and can predict production processes.

Acknowledgements. I am greatly indebted to I.J.R. Aitchison for extensive and illuminating comments. I am also grateful to Prof. G. Rupp and Prof. E. van Beveren for extensive discussions.

\section{References}

1. I.J.R. Aitchison, Nucl. Phys. A 189, 417 (1972)

2. K.M. Watson, Phys. Rev. 88, 1163 (1952)

3. B. Hyams et al., Nucl. Phys. B 64, 134 (1973)

4. R.H. Dalitz, S. Tuan, Ann. Phys. (New York) 10, 397 (1960)

5. D.V. Bugg, Eur. Phys. J. C 52, 55 (2007)

6. BES Collaboration, M. Ablikim et al., Phys. Lett. B 607, 243 (2005)

7. T.A. Lähde, U.G. Meissner, Phys. Rev. D 74, 034021 (2006)
8. BES Collaboration, M. Ablikim et al., Phys. Lett. B 598, 149 (2004)

9. A. Abele et al., Nucl. Phys. A 609, 562 (1996)

10. T. Åkessen et al., Nucl. Phys. B 264, 154 (1986)

11. R. Omnès, Nuovo Cim. 8, 316 (1958)

12. K.L. Au, D. Morgan, M.R. Pennington, Phys. Rev. D 35, 1633 (1987)

13. D.V. Bugg, J. Phys. G 34, 151 (2007)

14. D.V. Bugg, Eur. Phys. J. C 37, 433 (2004)

15. D.V. Bugg, Phys. Lett. B 632, 471 (2006)

16. W. Ochs, PhD thesis (University of Munich, 1974)

17. S. Pislak et al., Phys. Rev. D 57, 072004 (2003)

18. I. Caprini, G. Colangelo, H. Leutwyler, Phys. Rev. Lett. 96, 132001 (2006)

19. D.V. Bugg, Eur. Phys. J. C 47, 45 (2006)

20. V. Baru et al., Eur. Phys. J. A 23, 523 (2005)

21. Particle Data Group, J. Phys. G 33, 1 (2006)

22. A. Aloisio et al., Phys. Lett. B 537, 21 (2002)

23. BES Collaboration, M. Ablikim et al., Phys. Lett. B 603, 138 (2004)

24. I. Aitchison, private communication

25. M.G. Bowler et al., Nucl. Phys. B 97, 227 (1975)

26. I.J.R. Aitchison, M.G. Bowler, J. Phys. G 3, 1503 (1977)

27. J.L. Basdevant, E.L. Berger, Phys. Rev. D 16, 657 (1977)

28. G. Reifenrother, E. Klempt, Nucl. Phys. A 503, 886 (1989)

29. D. Morgan, M.R. Pennington, Phys. Rev. D 48, 1185 (1993)

30. D. Barberis et al., Phys. Lett. B 462, 462 (1999)

31. A.A. Carter, private communication

32. E. van Beveren, D.V. Bugg, F. Kleefeld, G. Rupp, Phys. Lett. B 641, 265 (2006)

33. A.V. Anisovich, V.V. Anisovich, A.V. Sarantsev, Z. Phys. A 359, 173 (1997)

34. G. Ascoli, H.W. Wyld, Phys. Rev. D 12, 43 (1975)

35. R.L. Schult, H.W. Wyld, Phys. Rev. D 16, 62 (1977) 